\section{Synthesis of Phosphorothioate Oligonucleotides with Stereodefined Phosphorothioate Linkages}

Phosphorothioate analogs of oligonucleotides (PS-oligos) constitute an important tool for studying the metabolism of nucleic acids (Eckstein, 2000, and references therein) and have been evaluated as potential therapeutics in the so-called "antisense" (Stein and Krieg, 1998) and "antigene" strategies (Thuong and Helene, 1993). In 1998, the U.S. Food and Drug Administration (FDA) approved the first PS-oligo, Fomirvirsen (trade name, Vitravene), for therapeutic application against cytomegalovirus (CMV) retinitis (Manoharan, 1999). Most of the second-generation antisense compounds that are currently undergoing clinical trials are PS-oligos (e.g., Isis Pharmaceuticals, Hybridon; Maier et al., 2000). PS-oligos are isoelectronic with natural oligonucleotides and, importantly, they are much more resistant towards intra- and extracellular nucleases (Wickstrom, 1986). These features are important with respect to their therapeutic applications. However, substitution of sulfur for one nonbridging oxygen in the internucleotide phosphate group induces asymmetry at the phosphorus atom, and standard chemical methods for the synthesis of oligo(deoxyribonucleoside phosphorothioate)s provide a mixture of $2^{n}$ diastereomers, where $n$ is the number of phosphorothioate linkages (Wilk and Stec, 1995). Therefore, even for relatively short PS-oligos (10- to 15-mers), thousands of diastereomers would be involved in interactions with other chiral biomolecules (e.g., DNA, RNA, or proteins) and, in principle, each diastereomer might interact in a slightly different manner.

The enzymatic synthesis of PS-oligos allows for the preparation of PS-oligonucleotides of $R_{\mathrm{P}}$-configuration at each phosphorothioate linkage (all- $R_{\mathrm{P}}$-PS-oligos) due to the stereoselectivity of all DNA and RNA polymerases identified to date (Hacia et al., 1994; Lackey and Patel, 1997; Tang et al., 1995). The first method for stereocontrolled chemical synthesis of PS-oligos, which was elaborated in the authors' laboratory (Stec et al., 1991), is based on a new chemistry employing $P$-diastereomerically pure nucleoside monomers possessing the 2-thio-1,3,2-oxathiaphospholane moiety attached to appropriately protected nucleosides at the $3^{\prime}$-O position (S.1; Fig. 4.17.1). Further studies resulted in the synthesis of monomers with the oxathiaphospholane ring substituted at position 4 with either two methyl groups (S.2; Stec et al., 1995) or a spiro pentamethylene ring (S.3; Stec et al., 1998). These substituents enhance a differentiation in chromatographic mobility of diastereomers, rendering their separation less laborious. The oxathiaphospholane monomers react in the presence of 1,8-diazabicyclo[5.4.0]undec-7-ene (DBU) with the $5^{\prime}$-OH group of a nucleoside (or growing oligonucleotide attached at the $3^{\prime}$ end to a DBU-resistant solid support) to yield a dinucleotide (or an elongated oligomer) with an internucleotide phosphorothioate diester bond, as depicted in Figure 4.17.2. The process is fully stereospecific and occurs with retention of configuration at the phosphorus atom. The chemical yield of the condensation process is not as efficient as that of the phosphoramidite or $H$-phosphonate methods (UNITS $3.3 \& 3.4$ ), but repetitive yields of $92 \%$ to 94\% allow syntheses of medium-sized oligomers (up to 15-mers). Longer oligonucleotides were obtained in poor yields and the syntheses were not reproducible. Oxathiaphospholanes that are ${ }^{18} \mathrm{O}$-labeled at the endocyclic position allowed for the synthesis of PS-oligos with internucleotide PS $\left[{ }^{18} \mathrm{O}\right]$-phosphorothioate moieties of predetermined chirality (Guga et al., 2001).
Synthesis of Modified Oligonucleotides and Conjugates

Contributed by Piotr Guga and Wojciech J. Stec

Current Protocols in Nucleic Acid Chemistry (2003) 4.17.1-4.17.28

Copyright $\odot 2003$ by John Wiley \& Sons, Inc.
4.17.1

Supplement 14 
For the synthesis of stereodefined PS-oligos via the oxathiaphospholane methodology presented in this unit, pure $P$-diastereomers of nucleoside oxathiaphospholane monomers are required. They are not commercially available, but can be efficiently obtained by phosphitylation of widely available $5^{\prime}-O$-DMTr- $N$-protected deoxyribonucleosides with oxathiaphospholane phosphitylating reagent followed by sulfurization. The methodology of their synthesis and use in solid-phase synthesis of PS-oligos is presented in consecutive protocols.

Basic Protocol 1 describes a detailed procedure for the synthesis of the phosphitylating reagent 2-chloro-spiro-4,4-pentamethylene-1,3,2-oxathiaphospholane. The procedure is general and may be applied to other analogs, depending on the aldehyde (or mercaptoalcohol) used.

Alternate Protocol 1 describes a procedure for synthesis of ${ }^{18} \mathrm{O}$-labeled mercaptoalcohol, which is used to synthesize labeled phosphitylating reagent and, subsequently, ${ }^{18} \mathrm{O}-\mathrm{la}-$ beled nucleoside monomers. These can be used for synthesis of stereodefined PS $\left[{ }^{18} \mathrm{O}\right]-$ oligos, which are useful compounds in studying the mechanism(s) of enzymatic reactions. Support Protocol 1 describes a method for transfer of dry solvents, required for this procedure.

Basic Protocol 2 outlines the synthesis of $5^{\prime}-O$-DMTr- $N$-protected-deoxyribonucleoside3'-O-(2-thio-spiro-4,4-pentamethylene-1,3,2-oxathiaphospholane)s (S.3) and their chromatographic separation into $P$-diastereomers. This method, although described for $\mathrm{dA}, \mathrm{dC}, \mathrm{dG}$, and $\mathrm{T}$ derivatives, can be also used for derivatizing other appropriately protected nucleosides. For example, in the authors' laboratory, $N^{6}$-benzoyl-7-deaza- $5^{\prime}-O$ (4,4'-dimethoxytrityl)-3'-O-(2-thio-spiro-4,4-pentamethylene-1,3,2-oxathiaphospholane)$2^{\prime}$-deoxyadenosine was obtained and separated into diastereomers (unpub.). Similarly, this method is suitable for phosphitylation of protected nucleosides with other oxathiaphospholane reagents containing different substituents, although the separation of diastereo-mers may be very difficult.

Alternate Protocol 2 describes the conversion of $5^{\prime}$-O-DMTr- $N$-protected-deoxyribonucleoside-3'-O-(2-thio-spiro-4,4-pentamethylene-1,3,2-oxathiaphospholane)s to their 2oxo-analogs with selenium dioxide. These monomers can be used to elongate stereodefined PS-oligos and generate segments of unmodified nucleotide units possessing phosphate internucleotide linkages. This goal cannot be achieved with the phosphoramidite or $H$-phosphonate methods, because the phosphorothioate linkages already

Synthesis of Phosphorothioate Oligonucleotides with Stereodefined Linkages

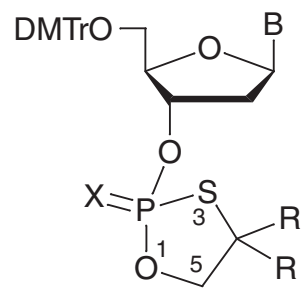

$$
\begin{aligned}
& 1 X=S, R=H \\
& 2 X=S, R=\mathrm{CH}_{3} \\
& 3 X=S, R, R=-\left(\mathrm{CH}_{2}\right)_{5^{-}} \\
& 4 X=O, R, R=-\left(\mathrm{CH}_{2}\right)_{5-}
\end{aligned}
$$

Figure 4.17.1 Structural features of deoxyribonucleoside oxathiaphospholane derivatives. Abbreviations: B, thymin-1-yl or N-protected nucleobase; DMTr, 4,4'-dimethoxytrityl. Adapted from Stec et al. (1998) with permission from the American Chemical Society. 

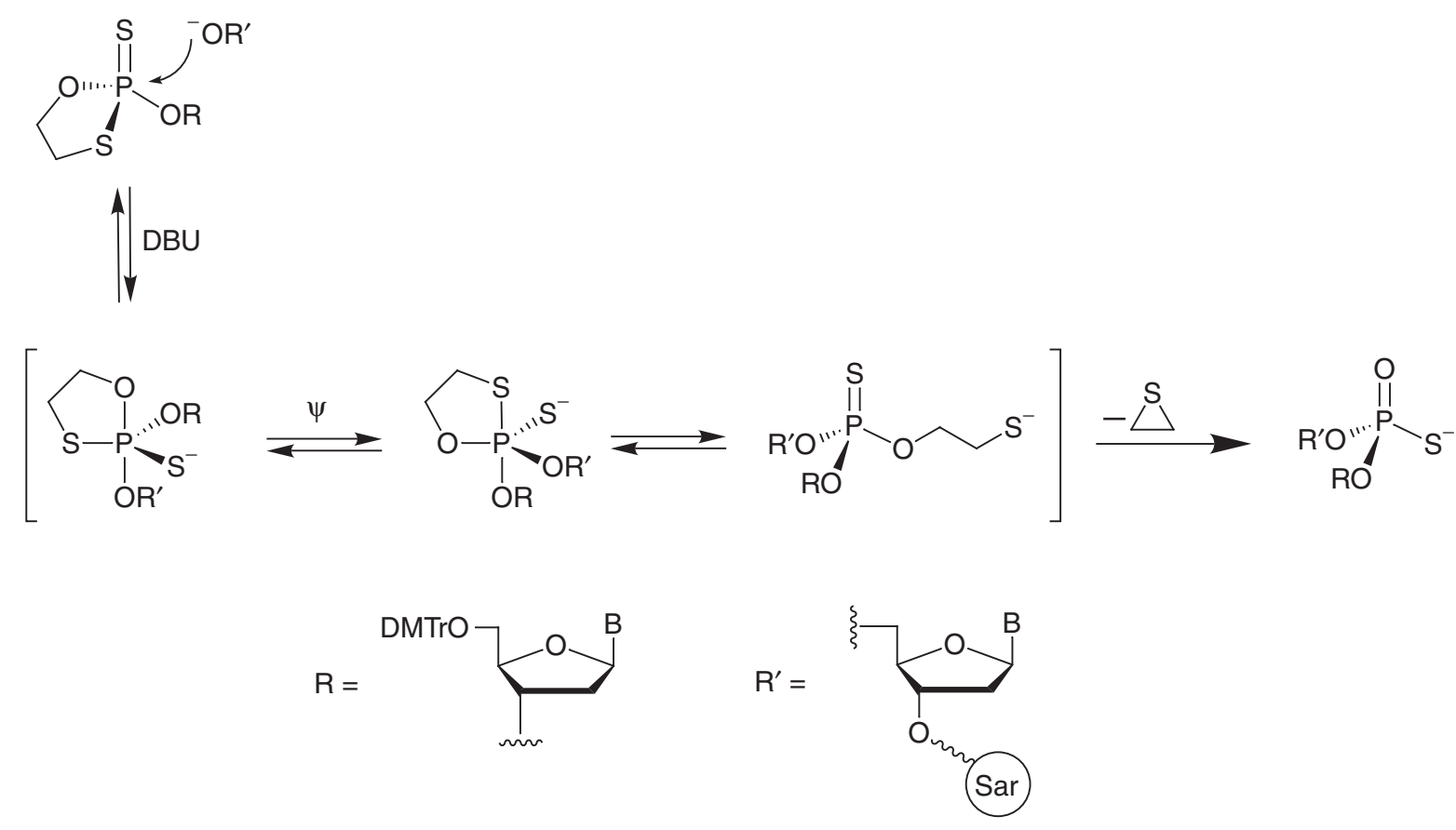

Figure 4.17.2 Mechanism of base-promoted oxathiaphospholane ring-opening condensation. Abbreviations: $\mathrm{B}$, thymin-1yl or N-protected nucleobase; DBU, 1,8-diazabicyclo[5.4.0]undec-7-ene; DMTr, 4,4'-dimethoxytrityl; $\psi$, pseudorotation; Sar, sarcosinylated or DBU-resistant solid support.

generated by the oxathiaphospholane method are diesters and would be oxidized in the $\mathrm{I}_{2} /$ water/pyridine routinely used for conversion of phosphites to phosphates.

Basic Protocol 3 outlines details of manual solid-phase synthesis of PS-oligos using oxathiaphospholane monomers. In principle, this synthesis can be performed on an automatic synthesizer, but the necessary modification of the manufacturer's protocols is impossible for the majority of synthesizers. The protocol for $1-\mu$ mol-scale automated solid-phase synthesis using an ABI 391 synthesizer (Applied Biosystems) has been published (Stec et al., 1998), but in many instances the software does not allow for any changes in the protocol. Also, the instrument should be able to deliver an additional solvent (methylene chloride) to the column in order to wash delivery lines after coupling. This is necessary to avoid formation of deposits inside the tubing and valves, which may lead to major failure of the instrument and expensive replacement of the clogged valve blocks. One also has to consider that, in using an automated synthesizer, significant amounts of monomer solutions are wasted during optimization of the protocol, and due to the dead volumes of the system. Therefore, manual synthesis of a limited number of oligomers may be economically more justified.

Support Protocol 2 describes preparation of solid supports for the synthesis of PS-oligos, which must be DBU-resistant because this strong base is necessary for the coupling step. This requirement is fulfilled by the use of Brown's sarcosinyl-succinoyl linker (Brown et al., 1989).
Synthesis of Modified Oligonucleotides and Conjugates

\subsection{3}

Supplement 14 

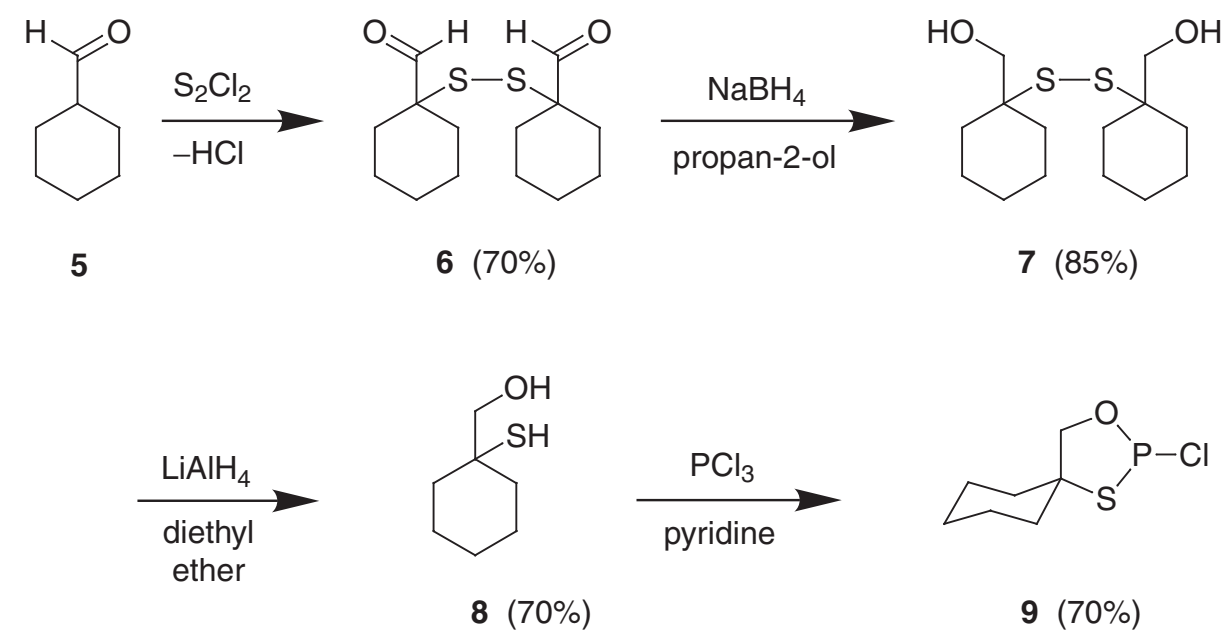

Figure 4.17.3 Synthesis of 2-chloro-spiro-4,4-pentamethylene-1,3,2-oxathiaphospholane (S.9) starting from cyclohexanecarboxaldehyde (S.5). Adapted from Stec et al. (1998) with permission from the American Chemical Society.

CAUTION: It is imperative that all reactions be run in a suitable fume hood with efficient ventilation. Many of the reactions in this unit are highly exothermic; safety glasses and reagent-impermeable protective gloves should be worn.

BASIC PROTOCOL 1

Synthesis of Phosphorothioate Oligonucleotides with Stereodefined Linkages

4.17.4

\section{SYNTHESIS OF PHOSPHITYLATING REAGENT: 2-CHLORO-spiro-4,4-PEN- TAMETHYLENE-1,3,2-OXATHIAPHOSPHOLANE}

The most simple oxathiaphosphitylating reagent, 2-chloro-1,3,2-oxathiaphospholane, can be obtained from the reaction of 2-mercaptoethanol with phosphorus trichloride in the presence of two molar equivalents of triethylamine (Martynov et al., 1969; Willson et al., 1975; Stec et al., 1991). Condensation of an appropriately protected 3'-OH-nucleoside with 2-chloro-1,3,2-oxathiaphospholane in pyridine solution, performed in the presence of dry elemental sulfur, provides nucleoside $3^{\prime}$ - $O$-(2-thiono-1,3,2-oxathiaphospholane)s (S.1; Fig. 4.17.1). However, their separation as $P$-diastereomers is very laborious and requires several consecutive silica gel chromatographic runs of partially enriched fractions. Therefore, it is recommended to synthesize 2-chloro-spiro-4,4-pentamethylene1,3,2-oxathiaphospholane (S.9) starting from cyclohexanecarboxaldehyde (S.5), as depicted in Figure 4.17.3. Using isobutyraldehyde in the same sequence of reactions, 2-chloro-4,4-dimethyl-1,3,2-oxathiaphospholane can be obtained. However, the phosphitylating reagent S.9, when used for synthesis of nucleotide monomers, provides much more useful compounds in terms of chromatographic separability as pure $P$-diastereomers. It is important to note that the fast-eluting isomers of 4,4-dimethyl- and 4,4-pentamethylene-oxathiaphospholane monomers are precursors of $R_{\mathrm{P}}$ internucleotidic phosphorothioate bonds. Conversely, analog internucleotide phosphorothioate bonds of $R_{\mathrm{P}}$ configuration are formed from the slow-eluting isomer of $\mathbf{S . 1}$.

Using this methodology, different analogs can be synthesized. However, it is important to obtain a phosphitylating reagent without additional centers of asymmetry, as the number of diastereomers will double with each new center, rendering separation of $P$-diastereomers very difficult or impossible. The relationship between chromatographic mobility and absolute configuration of the monomers must be checked for each new analog. 
4 to $5 \mathrm{M}$ and $1.5 \mathrm{M}$ sodium hydroxide $(\mathrm{NaOH})$

Sulfur monochloride $\left(\mathrm{S}_{2} \mathrm{Cl}_{2}\right)$, freshly distilled over $2 \mathrm{~g}$ elemental sulfur $\left(\mathrm{S}_{8}\right.$; dried $\geq 12 \mathrm{hr}$ under vacuum) per $50 \mathrm{~mL} \mathrm{~S} \mathrm{Sl}_{2}$

Argon (or, optionally, nitrogen), dry

Cyclohexanecarboxaldehyde (Fluka)

Methylene chloride

Diethyl ether, anhydrous

Sodium borohydride $\left(\mathrm{NaBH}_{4}\right)$

Isopropyl alcohol

Anti-bumping granules

$20 \%$ (w/v) hydrochloric acid

Chloroform

Magnesium sulfate, anhydrous

Hexane

Lithium aluminum hydride

Ethyl acetate, dry

Tetrahydrofuran (THF), with traces of added moisture

$10 \%$ (v/v) $\mathrm{H}_{2} \mathrm{O} / \mathrm{THF}$

Phosphorus trichloride $\left(\mathrm{PCl}_{3}\right)$

Benzene, anhydrous

Pyridine

Dry molecular sieves (4A, 4- to 6- $\mu$ m-o.d. beads, Aldrich)

250-mL absorber with safety flask (see Fig. 4.17.4)

250-mL four-neck round-bottom flask

Heated oil bath capable of magnetic stirring

Thermometer (capable of reading $150^{\circ} \mathrm{C}$ )

100 -mL dropping funnel

Reflux condensers

Glass gas inlet adapter (preferred) or syringe needle and rubber septum

Rotary evaporator with a water aspirator and a diaphragm vacuum pump (10 to 15 $\mathrm{mmHg}$; optional)

500-mL Erlenmeyer flask (29/42 joint)

1-L two-neck round-bottom flask (two 29/42 joints)

Stopcock, 29/42

Flexible adapter (glass M/F joints, 29/42, on corrugated Teflon tubing; optional)

500-mL separatory funnel

Filter funnel and Whatman no.1 filter paper (or equivalent)

High-vacuum fractional distillation apparatus

High-vacuum oil pump $(0.01 \mathrm{mmHg})$

NOTE: Upon storage, cyclohexanecarboxaldehyde undergoes polymerization. Order only the amount required for use within 2 to 3 weeks.

NOTE: Within this unit, evaporation of solvents is performed using a rotary evaporator connected to a water aspirator, unless otherwise specified.

Synthesis of

Modified

Oligonucleotides

and Conjugates

4.17.5

Supplement 14 


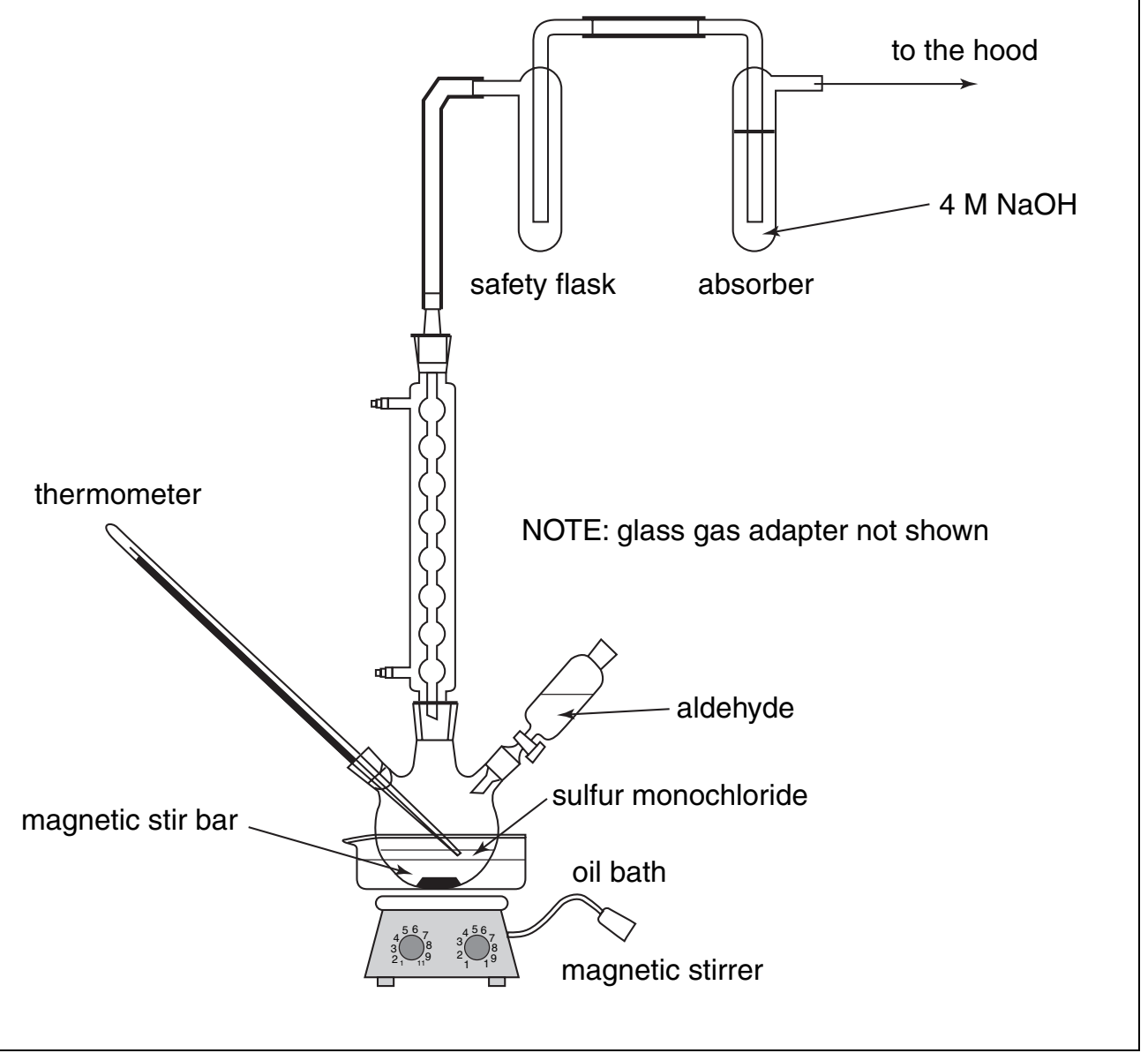

Figure 4.17.4 System assembly for synthesis of S.6. The glass gas adapter (or septum and needle) for delivery of dry argon should be mounted in the fourth neck of the flask (not shown).

\section{Synthesize 2,2'-dithiobis(cyclohexanecarbaldehyde) (S.6)}

1. Prepare a $250-\mathrm{mL}$ absorber containing $\sim 150 \mathrm{~mL}$ of 4 to $5 \mathrm{M} \mathrm{NaOH}$ (see Fig. 4.17.4).

2. Assemble a reactor consisting of a $250-\mathrm{mL}$ four-neck, round-bottom flask (to be heated in an oil bath with magnetic stirring) equipped with a thermometer, a 100-mL dropping funnel, a condenser, and a magnetic stir bar. Connect the outlet of the condenser via a safety flask to the absorber and be sure that the absorber vents into the hood.

The capacity of the safety flask must be sufficient to accommodate the solution of sodium hydroxide from the absorber.

3. Add $30 \mathrm{~g}(17.8 \mathrm{~mL}, 0.22 \mathrm{~mol})$ of freshly distilled sulfur monochloride to the flask. Make sure that the thermometer is in contact with the liquid. Deliver dry argon close to the bottom of the flask through the fourth joint either with a glass gas inlet adapter or with a syringe needle inserted through a rubber septum.

Since gaseous hydrogen chloride is liberated from the reaction, the use of a glass inlet adapter, rather than a syringe needle, is recommended.

Synthesis of Phosphorothioate Oligonucleotides with Stereodefined Linkages

4. Apply heating until sulfur monochloride reaches $60^{\circ} \mathrm{C}$. Stabilize the temperature.

5. Add, dropwise through the dropping funnel, $50 \mathrm{~g}(56 \mathrm{~mL}, 0.44 \mathrm{~mol})$ of cyclohexanecarboxaldehyde over a 60 -min period with stirring, keeping the temperature at $60^{\circ} \mathrm{C}$. Remove the oil bath and allow the mixture to continue stirring another $10 \mathrm{~min}$. 
6. Stop stirring and remove the stir bar. Leave the reaction mixture until it reaches room temperature and solidifies $(\sim 1 \mathrm{hr})$.

7. Dissolve the solid residue in $150 \mathrm{~mL}$ of methylene chloride, then evaporate to dryness using a rotary evaporator connected to a water aspirator.

CAUTION: During evaporation gaseous hydrogen chloride is liberated.

8. Add $150 \mathrm{~mL}$ of diethyl ether and gently reflux until the solid residue is dissolved. Transfer the solution into a 500-mL Erlenmeyer flask and close the stopcock. Cool down the mixture (containing the synthesized S.6) and keep overnight in a refrigerator $\left(4^{\circ} \mathrm{C}\right)$ to allow crystallization. Collect crystalline $\mathbf{S . 6}$ by filtration.

Approximately $35 \mathrm{~g}$ of 2,2'-dithiobis(cyclohexanecarbaldehyde) (S.6) should be collected as a white solid ( $70 \%$ yield, m.p. $88^{\circ}$ to $\left.89^{\circ} \mathrm{C}\right) .{ }^{l} \mathrm{H} \mathrm{NMR}\left(\mathrm{CDCl}_{3}, \delta\right): 8.98 \mathrm{ppm}(\mathrm{s}, 1 \mathrm{H}$, $\mathrm{CHO}$ ), 1.2-2.1 ppm (m, 1OH). FAB MS (positive mode, $\mathrm{Cs}^{+}, 13 \mathrm{keV}$, matrix $\mathrm{NBA}$ ) $\mathrm{m} / \mathrm{z} 286$, $\left[\mathrm{M}^{+}, 25 \% ; \mathrm{m} / \mathrm{z} 111,\left[\mathrm{C}_{6} \mathrm{H}_{10} \mathrm{CHO}^{+}, 100 \%\right.\right.$.

\section{Synthesize 2,2'-dithiobis(cyclohexanemethanol) (S.7)}

9. In a 1-L two-neck flask (two 29/42 joints), equipped with a reflux condenser and a stopcock, suspend $5.67 \mathrm{~g}(0.15 \mathrm{~mol})$ of $\mathrm{NaBH}_{4}$ in $500 \mathrm{~mL}$ isopropyl alcohol. Add anti-bumping granules and heat to boiling.

10. While gently refluxing, remove the stopcock momentarily and add, with a chemical spoon, $\sim 2 \mathrm{~g}$ of $\mathbf{S . 6}$ every 3 to $5 \mathrm{~min}$ in 10 to 12 portions for a total of $21.5 \mathrm{~g}(0.075$ mol).

CAUTION: The addition of each portion of 2,2'-dithiobis(cyclohexanecarboxaldehyde) results in enhanced boiling and emission of vapors of isopropyl alcohol through the open neck. The stopcock should thus be closed as soon and possible. Alternatively, one can use a flexible adapter (glass M/F joints on corrugated Teflon tubing) for stepwise delivery of $2,2^{\prime}$-dithiobis(cyclohexanecarboxaldehyde) without opening the reactor.

11. Reflux the mixture for $1 \mathrm{hr}$, then evaporate to dryness and add $200 \mathrm{~mL}$ of $1.5 \mathrm{M}$ sodium hydroxide.

12. Cautiously neutralize the mixture with $20 \%$ hydrochloric acid, checking pH with indicator strips.

13. Transfer the mixture to a $500-\mathrm{mL}$ separatory funnel and extract the solution twice, each time with $150 \mathrm{~mL}$ chloroform. Dry the organic layer with anhydrous magnesium sulfate and evaporate the solvent.

14. Dissolve the product in $200 \mathrm{~mL}$ of diethyl ether and add, with stirring, a few 3- to 5-mL aliquots of hexane until the mixture becomes translucent. Leave in a refrigerator $\left(4^{\circ} \mathrm{C}\right)$ overnight for crystallization. Collect crystalline $\mathbf{S . 7}$ by filtration.

Approximately $19 \mathrm{~g}$ of 2,2'-dithiobis(cyclohexanemethanol) (S.7) should be collected as a colorless crystalline material $\left(85 \%\right.$ to $88 \%$ yield, m.p. $49^{\circ}$ to $\left.50^{\circ} \mathrm{C}\right) .{ }^{l} \mathrm{H} \mathrm{NMR}\left(\mathrm{CDCl}_{3}, \delta\right)$ : $3.54 \mathrm{ppm}\left(\mathrm{s}, 2 \mathrm{H}, \mathrm{CH}_{2} \mathrm{OH}\right), 2.23 \mathrm{ppm}\left(\mathrm{s}, 1 \mathrm{H}, \mathrm{CH}_{2} \mathrm{OH}\right), 1.2-1.8 \mathrm{ppm}(\mathrm{m}, \mathrm{lOH}) .{ }^{13} \mathrm{C} \mathrm{NMR}$ $\left(\mathrm{CDCl}_{3}, \delta\right): 21.9,25.73,32.45,56.18,67.99 \mathrm{ppm}$. FAB MS (positive mode, $\mathrm{Cs}^{+}, 13 \mathrm{keV}$, matrix NBA) $\mathrm{m} / \mathrm{z} 290,\left[\mathrm{MJ}^{+}, 45 \% ; \mathrm{m} / \mathrm{z} 273,[\mathrm{M}-\mathrm{OH}]^{+}, 25 \% ; \mathrm{m} / \mathrm{z} 113,\left[\mathrm{C}_{6} \mathrm{H}_{10} \mathrm{CH}_{2} \mathrm{OH}\right]^{+}\right.$, $100 \%$.

\section{Synthesize 2-mercaptocyclohexanemethanol (S.8)}

15. In a 1-L two-neck flask equipped with a reflux condenser and a dropping funnel (atmosphere of dry argon or nitrogen) suspend $5.9 \mathrm{~g}(0.16 \mathrm{~mol})$ lithium aluminum hydride in $500 \mathrm{~mL}$ of dry diethyl ether.

CAUTION: The suspension of lithium aluminum hydride in diethyl ether is highly flammable. Advise coworkers of the hazard and keep an appropriate fire extinguisher at hand.

Synthesis of Modified Oligonucleotides and Conjugates

4.17.7

Supplement 14 
16. Add dropwise through the dropping funnel a solution of $4.6 \mathrm{~g}(0.16 \mathrm{~mol}) \mathrm{S} .7$ in 150 $\mathrm{mL}$ of diethyl ether over $60 \mathrm{~min}$ with magnetic stirring. Continue stirring for an additional $60 \mathrm{~min}$.

The reaction is exothermic and mild reflux occurs.

17. Cautiously decompose excess reducing agent by adding dropwise through the funnel $3 \mathrm{~mL}$ of dry ethyl acetate, followed by $10 \mathrm{~mL}$ THF containing traces of moisture, and then $\sim 10 \mathrm{~mL}$ of $10 \%$ water/THF, until the solid suspension becomes gray and finally white.

18. Filter off inorganic salts using a filter funnel and Whatman no. 1 filter paper, and dry the filtrate over anhydrous magnesium sulfate. Filter off the drying agent and evaporate the filtrate to dryness.

19. Distill the residue in a high-vacuum fractional distillation apparatus under reduced pressure $(0.05 \mathrm{mmHg}$, provided by high-vacuum oil pump). Collect the fraction boiling between $74^{\circ}$ and $76^{\circ} \mathrm{C}$, which contains $\mathbf{S . 8}$.

CAUTION: Avoid overheating the vessel. Keep pressure below $0.1 \mathrm{mmHg}$.

Approximately $3.2 \mathrm{~g}$ of 2-mercaptocyclohexanemethanol (S.8) should be collected as a colorless oil ( $70 \%$ yield, $\left.n_{D}{ }^{20}=1.5188\right) .{ }^{l} \mathrm{H} \mathrm{NMR}\left(\mathrm{CDCl}_{3}, \delta\right): 3.49 \mathrm{ppm}\left(\mathrm{s}, 2 \mathrm{H}, \mathrm{CH}_{2} \mathrm{OH}\right)$, $2.15 \mathrm{ppm}$ (bs, $\left.1 \mathrm{H}, \mathrm{CH}_{2} \mathrm{OH}\right), 1.31 \mathrm{ppm}(\mathrm{s}, 1 \mathrm{H}, \mathrm{CHSH}), 1.15-1.85 \mathrm{ppm}(\mathrm{m}, 10 \mathrm{H}) .{ }^{13} \mathrm{C} \mathrm{NMR}$ $\left(\mathrm{CDCl}_{3}, \delta\right.$ ): 22.0, 26.07, 36.06, 52.34, 73.12 ppm. FAB MS (negative mode, $\mathrm{Cs}^{+}, 13 \mathrm{keV}$, matrix GLY) $\mathrm{m} / \mathrm{z}$ 145, $\left[\mathrm{MJ}^{-}, 100\right.$. FAB MS (positive mode, $\mathrm{Cs}^{+}, 13 \mathrm{keV}$, matrix GLY) $\mathrm{m} / \mathrm{z}$ 113, $\left[\mathrm{C}_{6} \mathrm{H}_{10} \mathrm{CH}_{2} \mathrm{OH}\right]^{+}, 45 \% ; \mathrm{m} / 2 \mathrm{129,}\left[\mathrm{C}_{6} \mathrm{H}_{10} \mathrm{SHCH}_{2}\right]^{+}, 20 \%$.

\section{Synthesize 2-chloro-spiro-4,4-pentamethylene-1,3,2-oxathiaphospholane (S.9)}

20. In a 1-L two-neck flask equipped with a thermometer and a dropping funnel, add 28.2 $\mathrm{g}(0.21 \mathrm{~mol}) \mathrm{PCl}_{3}$ to $500 \mathrm{~mL}$ of dry benzene under an argon (optionally nitrogen) atmosphere. Cool the flask to $5^{\circ} \mathrm{C}$ with an ice bath. Add, dropwise through the funnel, a solution of $20 \mathrm{~g}(0.14 \mathrm{~mol}) \mathbf{S . 8}$ and $22 \mathrm{~mL}(0.27 \mathrm{~mol})$ pyridine in $35 \mathrm{~mL}$ dry benzene over a 15-min period with magnetic stirring. Keep the temperature of the reaction mixture below $10^{\circ} \mathrm{C}$.

21. Continue stirring at room temperature for $30 \mathrm{~min}$ and filter off pyridine hydrochloride with exclusion of moisture. Load the reaction mixture in a filter funnel inside a bag filled with dry argon (or nitrogen) and gently apply suction to keep the bag slightly inflated with continuous delivery of dry gas.

22. Evaporate the solvent under reduced pressure with exclusion of moisture (preferably in a rotary evaporator equipped with a diaphragm vacuum).

If a water aspirator must be used, insert a drying tube filled with blue indicator silica gel between the rotary evaporator and the aspirator to reduce the risk of hydrolysis of the product. Apply vacuum gently.

23. Distill the product in a high-vacuum fractional distillation apparatus under reduced pressure $(0.01 \mathrm{mmHg}$, provided by a high-vacuum oil pump). Collect the fraction boiling between $82^{\circ}$ and $84^{\circ} \mathrm{C}$, which contains $\mathbf{S . 9}$.

CAUTION: Avoid overheating the vessel. Keep pressure at $0.01 \mathrm{mmHg}$. When overheated, spontaneous decomposition of the crude product may occur, leading to destruction of the apparatus.

Approximately $20 \mathrm{~g}$ of 2-chloro-spiro-4,4-pentamethylene-1,3,2-oxathiaphospholane (S.9) should be collected as a colorless liquid (70\% to $75 \%$ yield). ${ }^{31} P$ NMR $\left(C_{6} D_{6}\right.$; $\left.\delta\right) 217.7$ ppm; EI (electron impact) MS: $(70 \mathrm{eV}) \mathrm{m} / 2.210,\left[\mathrm{MJ}^{+}, 12 \% ; \mathrm{m} / \mathrm{z}, 175,[\mathrm{M}-\mathrm{Cl}]^{+}, 5.8 \% ; \mathrm{m} / \mathrm{z}\right.$ $90,100 \%$.

24. Store $\mathbf{S . 9}$ in a tightly closed vessel inside another tightly closed container filled with several grams of dry $4 \mathrm{~A}$ molecular sieves at $-20^{\circ} \mathrm{C}$ (stable for at least 1 year).

4.17.8 
SYNTHESIS OF $2,2^{\prime}$-DITHIOBIS( $\left(\left[{ }^{18}\right.\right.$ O]CYCLOHEXANECARBOXALDEHYDE)

The ${ }^{18} \mathrm{O}$-labeled 2,2'-dithiobis(cyclohexanecarboxaldehyde), which can be further transformed into the corresponding phosphitylating reagent as described in Basic Protocol 1, is obtained by hydrolysis of the $N$-phenylimine derivative of 2,2'-dithiobis(cyclohexanecarboxaldehyde) with $\mathrm{H}_{2}\left[{ }^{18} \mathrm{O}\right]$, catalyzed with gaseous hydrogen chloride (Fig. 4.17.5). The $N$-phenylimine derivative is obtained from 2,2'-dithiobis(cyclohexanecarboxaldehyde) (S.6; see Basic Protocol 1, step 8) upon treatment with aniline.

Additional Materials (also see Basic Protocol 1)

2,2'-Dithiobis(cyclohexanecarboxaldehyde) (S.6; see Basic Protocol 1, step 8)

Aniline, freshly distilled in inert atmosphere

95:5 (v/v) chloroform/hexane

$\mathrm{H}_{2}\left[{ }^{18} \mathrm{O}\right](95$ atom\%)

Hydrogen chloride, anhydrous

Tetrahydrofuran (THF), dried over sodium hydride

250-mL two-neck round-bottom flasks

Azeotropic trap (e.g., mini Dean-Stark trap, Aldrich)

$8 \times 40-\mathrm{cm}$ chromatography column packed with silica gel 60, 230 to 400 mesh (Merck)

Rubber septum

TLC silica gel plates with UV indicator (Merck; also see APPENDIX $3 D$ )

High vacuum valve (e.g., Rotaflo, Quickfit)

Drying tube $(8 \times 5 / 8$ in. with connectors, Aldrich)

2- to 5-mL gas-tight syringe

Rotary evaporator with water aspirator or membrane pump

Buchner funnel with glass frit

Additional reagents and equipment for column chromatography (APPENDIX $3 E$ ), thin-layer chromatography (TLC; APPENDIX $3 D$ ), and high-vacuum transfer of solvent (see Support Protocol 1)

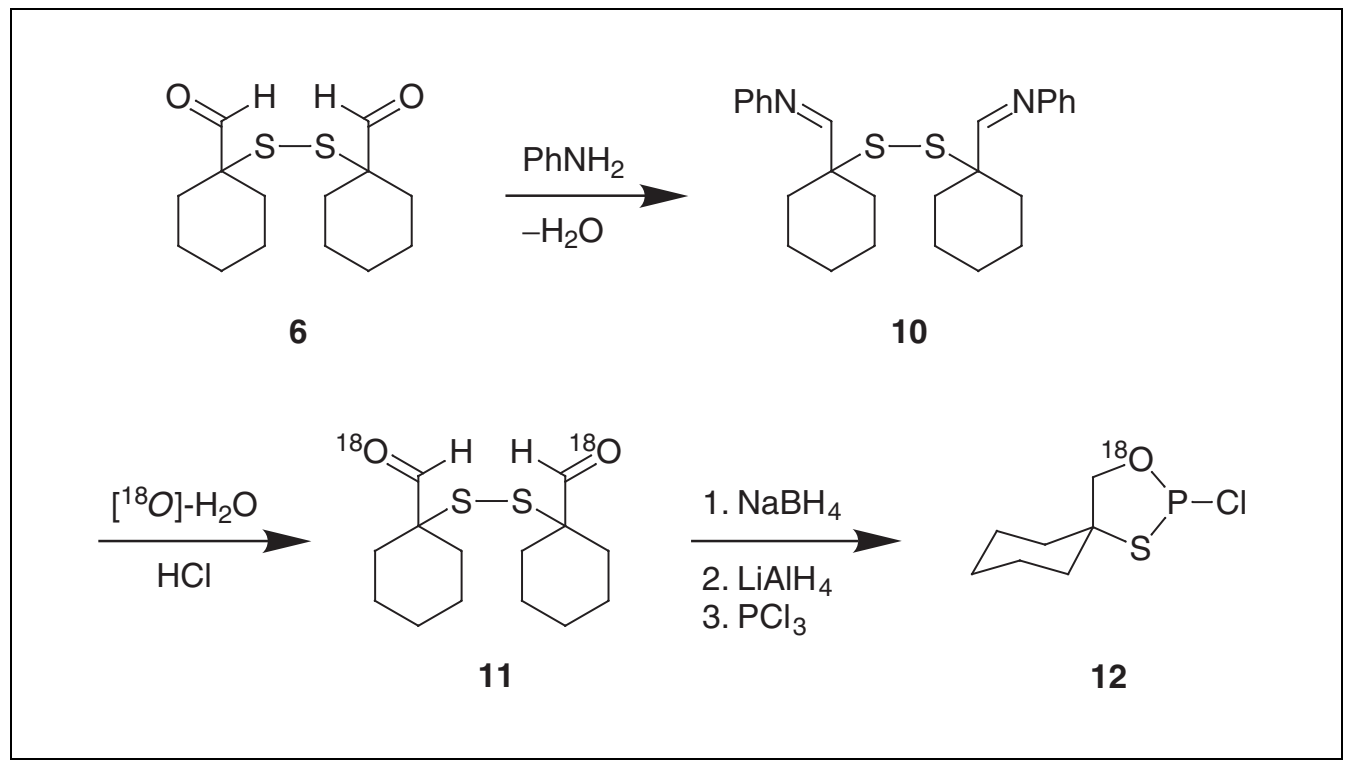

Figure 4.17.5 Synthesis of 2-chloro-spiro-4,4-pentamethylene-1,3,2-[ $\left.{ }^{18} \mathrm{O}\right]$ oxathiaphospholane (S.12) starting from 2,2'-dithiobis(cyclohexanecarboxaldehyde) (S.6).
Synthesis of Modified Oligonucleotides and Conjugates

4.17.9

Supplement 15 
1. In a 250-mL two-neck flask equipped with an azeotropic trap, reflux condenser, and dropping funnel, dissolve $14.3 \mathrm{~g} \mathrm{(0.05} \mathrm{mol)} \mathrm{of} \mathrm{2,2'-dithiobis(cyclohexanecarboxal-}$ dehyde) (S.6) in $150 \mathrm{~mL}$ benzene. Add anti-bumping granules and heat to boiling.

2. Add, dropwise through the dropping funnel, a solution of $10.0 \mathrm{~mL}(10.2 \mathrm{~g}, 0.11 \mathrm{~mol})$ aniline in $25 \mathrm{~mL}$ benzene over a 30-min period. Continue the reaction for $30 \mathrm{~min}$, keeping gently boiling with azeotropic removal of liberated water.

The end of the reaction is confirmed by disappearance of a resonance line of the aldehyde proton in the ${ }^{1} H$ NMR spectrum.

3. Cool the reaction mixture to room temperature and evaporate the solvent under reduced pressure using a rotary evaporator with a water aspirator.

4. Dissolve the residue in 15 to $20 \mathrm{~mL}$ of benzene and apply to an $8 \times 40-\mathrm{cm}$ chromatography column packed with $\sim 200 \mathrm{~g}$ of 230 to 400 mesh silica gel.

5. Elute the column with chloroform and collect the eluate in $12-$ to $15-\mathrm{mL}$ fractions.

6. Analyze fractions by TLC on silica gel plates (APPENDIX 3D). Develop TLC plates with 95:5 (v/v) chloroform/hexane.

7. Combine all fractions that contain the desired product $\left(\mathbf{S . 1 0} ; R_{\mathrm{f}}=0.55\right)$. Evaporate the solvent under reduced pressure.

Typically $18 \mathrm{~g}$ (80\% yield) of the N-phenylimine derivative (S.10; see Fig. 4.17.5) should be obtained. ${ }^{1} \mathrm{H} \mathrm{NMR}\left(\mathrm{CDCl}_{3}\right.$, $\left.\delta\right): 6.67-6.80 \mathrm{ppm}(\mathrm{m}, \mathrm{lH}), 7.07-7.55 \mathrm{ppm}(\mathrm{m}, 4 \mathrm{H}), 3.6 \mathrm{ppm}$ (very broad singlet, $1 \mathrm{H}, \mathrm{CH}=\mathrm{NPh}), 1.29-2.18 \mathrm{ppm}(\mathrm{m}, 10 \mathrm{H}) .{ }^{13} \mathrm{C} \mathrm{NMR}\left(\mathrm{CDCl}_{3}, \mathrm{\delta}\right): 22.8$, $24.88,25.22,30.05,30.15,33.23,56.13,56.793,60.13,60.62,76.38,77.01,77.65,114.84$, $118.14,120.63,120.69,125.42,125.69,128.11,128.78,129.00,146.28,150.99,151.33$, $164.78,165.62,194.09,194.77$. This number of resonances in the ${ }^{13} \mathrm{C} N M R$ spectrum reflects the presence of cis- and trans-isomers of the $N$-phenylimine derivative.

\section{Synthesize labeled 2,2'-dithiobis $\left(\left[^{18}\right.\right.$ O $]$ cyclohexanecarboxaldehyde) (S.11)}

8. Place $16 \mathrm{~g}(0.037 \mathrm{~mol}) \mathbf{S . 1 0}$ in a $250-\mathrm{mL}$ two-neck round-bottom flask with a magnetic stir bar inside, with a high-vacuum valve in one joint and a rubber septum in the other, and dry overnight at high vacuum $(0.01 \mathrm{mmHg}$; provided by high-vacuum oil pump). At the end of drying, deliver dry argon gas to the flask through the septum.

9. Prepare an absorber containing $\sim 150 \mathrm{~mL}$ of 4 to $5 \mathrm{M} \mathrm{NaOH}$ (see Basic Protocol 1, step 1).

10. Using the vacuum line technique (see Support Protocol 1), transfer $\sim 100 \mathrm{~mL}$ of dry THF to the flask containing $\mathbf{S . 1 0}$.

11. Connect the vacuum valve with the absorber through an $8 \times 5 / 8-$ in. drying tube (filled with anhydrous magnesium sulfate) and a safety flask (Fig. 4.17.4).

12. Add $1.8 \mathrm{~mL}(0.9 \mathrm{~mol}) \mathrm{H}_{2}\left[{ }^{18} \mathrm{O}\right]$ (20\% excess) with a 2- to 5-mL gas-tight syringe.

13. Flush the apparatus continously with dry argon and cool the mixture to $<5^{\circ} \mathrm{C}$ in an ice bath.

14. Remove the septum and quickly install a glass gas inlet adapter for delivery of hydrogen chloride. Adjust the adapter so that its end is $\sim 1.5$ to $2 \mathrm{~cm}$ above the level of the liquid.

Synthesis of Phosphorothioate Oligonucleotides with Stereodefined Linkages

4.17.10
15. Slowly deliver anhydrous hydrogen chloride with magnetic stirring of the mixture.

\section{Precipitation of anilinium hydrochloride should be observed during this process.}


16. Continue hydrolysis until the reaction mixture becomes pale green-yellow. Remove ice bath and allow the mixture to reach room temperature.

17. Filter off the precipitate using a Buchner funnel. Wash twice with 20 to $30 \mathrm{~mL}$ THF.

18. Combine filtrates and evaporate solvent under reduced pressure.

CAUTION: The THF vapor will be strongly acidic, as it is saturated with hydrogen chloride.

19. Dissolve the residue in $100 \mathrm{~mL}$ of THF and evaporate the solvent again.

20. Dissolve the residue in diethyl ether and crystallize by cooling (see Basic Protocol 1 , step 8).

21. Analyze the product using electron impact (EI) mass spectrometry.

Each aldehyde group of the final product is isotopically labeled to the extent of $87 \%$. The spectrum should contain three peaks at $\mathrm{m} / \mathrm{z} 286,288$, and 290, corresponding to the unlabeled, singly labeled, and doubly labeled compounds, respectively. The intensities of the ions are 1.7\%, 22.6\%, and 75.7\%, respectively. The isotope content remains unchanged during subsequent reactions leading to the final phosphitylating reagent.

22. Continue the synthesis (see Basic Protocol 1, starting from step 9).

\section{HIGH-VACUUM TECHNIQUE FOR TRANSFER OF DRY SOLVENTS}

This technique allows for transfer of dry solvents from a reservoir (where the solvent is stored over a drying reagent such as sodium hydride) into a reaction flask with exclusion of moisture. For successful transfer, the quality of vacuum valves is absolutely essential. Figure 4.17.6 shows principal construction of the apparatus, which can be assembled from generic glassware. All components must be tested to make sure that they are safe for high-vacuum usage (i.e., at $<0.5 \mathrm{mmHg}$ ).

1. Set up the apparatus as shown in Fig. 4.17.6, but leave the flask containing THF over $\mathrm{NaH}$ not yet immersed in the warm water bath and the receiving flask not yet immersed in the dry ice/isopropanol bath.

2. Close high-vacuum valves $\mathrm{A}$ and $\mathrm{B}$.

3. Attach the apparatus to high vacuum $(<0.5 \mathrm{mmHg})$.

4. Open valve B for several seconds.

5. Close valve $\mathrm{B}$.

6. Open valve A for several seconds. If gentle boiling of THF is observed, go to step 9.

7. Close valve $\mathrm{A}$.

8. Decrease the pressure in the system by repeating steps 4 to 7 .

9. While valve B is closed, immerse the receiving flask in the dry ice/isopropanol bath.

10. Immerse the reservoir in the warm water bath $\left(\sim 50^{\circ}\right.$ to $\left.55^{\circ} \mathrm{C}\right)$. Alternatively, use a hair dryer to provide heat to the reservoir.

11. After the required volume of solvent has been transferred, stop heating and remove the dry ice bath. Allow both flasks to reach ambient temperature.

12. Disconnect the apparatus from the high vacuum and attach the outlet to a line with dry argon.

Synthesis of Modified Oligonucleotides and Conjugates

4.17.11

Supplement 15 


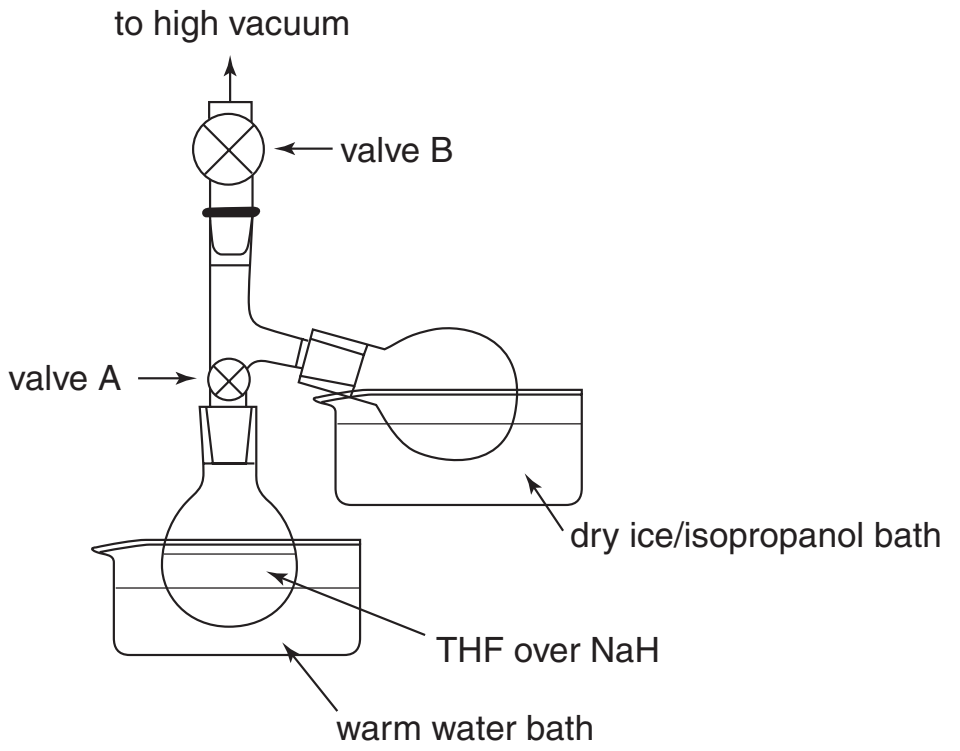

Figure 4.17.6 Apparatus for high-vacuum transfer of dry solvents.

13. Open valve B and fill the system with argon.

14. Close valves A and B.

15. Disconnect the flask with transferred solvent and close immediately with a stopcock or septum.

BASIC PROTOCOL 2

Synthesis of Phosphorothioate Oligonucleotides with Stereodefined Linkages

4.17.12

\section{SYNTHESIS OF 5' $O$-DMTr-DEOXYRIBONUCLEOSIDE-3'- $O$-(2-THIO-4,4- spiro-PENTAMETHYLENE-1,3,2-OXATHIAPHOSPHOLANE)S AND THEIR SEPARATION INTO $P$-DIASTEREOMERS}

The oxathiaphospholane method of stereocontrolled synthesis of PS-oligos, depicted in Figure 4.17.2, is based on availability of diastereomerically pure oxathiaphospholane monomers S.1-S.3. Separation of monomers $\mathbf{S . 1}$ and $\mathbf{S . 2}$ as pure $P$-diastereomers requires tedious silica gel column chromatography or costly preparative HPLC. Monomer S.3, obtained by introducing a pentamethylene substituent at position 4 of the oxathiaphospholane ring, possesses a satisfactory separability of diastereomers. Appropriate $5^{\prime}-O$ DMTr- $N$-protected deoxyribonucleosides are phosphitylated at room temperature with S.9 or S.12 in acetonitrile, in the presence of diisopropylethylamine, to yield the corresponding phosphites, which are further sulfurized with elemental sulfur. Silica gel column chromatography affords $\mathbf{S . 3}$ (or its ${ }^{18} \mathrm{O}$-labeled analog) as a diastereomeric mixture in satisfactory yield (75\% to $85 \%$ ). The deoxyguanosine derivative of $\mathbf{S . 3}$ should be additionally protected at $\mathrm{O} 6$ with diphenylcarbamoyl chloride to improve the yield of the condensation step. Without this protection, the repetitive yield of condensation of the deoxyguanosine oxathiaphospholane monomer drops below 90\%. The S.3 monomers (B $\left.=\mathrm{T}, \mathrm{A}^{\mathrm{Bz}}, \mathrm{C}^{\mathrm{Bz}}, \mathrm{G}^{i \mathrm{Bu}, \mathrm{DPC}}\right)$ are separated by column chromatography into fast- and sloweluting species. The stereochemistry of the coupling has been checked for each of 32 combinations of diastereomeric dinucleotides $\mathrm{N}_{\mathrm{PS}} \mathrm{N}(\mathrm{N}=\mathrm{dG}, \mathrm{dA}, \mathrm{dC}, \mathrm{T})$. All four fast-eluting diastereomers of $\mathbf{S . 3}$ are precursors of the dinucleoside $3^{\prime}, 5^{\prime}$-phosphorothioates of $\mathrm{R}_{\mathrm{P}}$ configuration. Slow-eluting isomers of $\mathbf{S . 3}$ yield phosphorothioate linkages of $S_{P}$ configuration. 
NOTE: It is important to use acid-free eluents for all silica gel chromatography of the monomers to avoid partial detritylation of the products. This can be done by distillation of chloroform with $1 \mathrm{~mL}$ pyridine, and then by adding 2 to $3 \mathrm{~mL}$ pyridine per liter of all eluents (chloroform and mixtures of ethyl acetate, butyl acetate, and benzene).

\section{Materials}

$5^{\prime}-O$-DMTr- $N$-protected deoxyribonucleosides (Chemgenes):

$N^{6}$-Benzoyl-5'-O-(4,4'-dimethoxytrityl)-2'-deoxyadenosine $\left(5^{\prime}-O\right.$-DMTr-dA $\left.{ }^{\mathrm{Bz}}\right)$

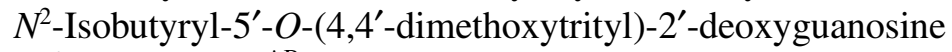
$\left(5^{\prime}-O-\mathrm{DMTr}-\mathrm{dG}^{i-\mathrm{Bu}}\right)$

$N^{4}$-Benzoyl-5'-O-(4,4'-dimethoxytrityl)-2'-deoxycytidine $\left(5^{\prime}-O-\mathrm{DMTr}-\mathrm{dC}{ }^{\mathrm{Bz}}\right)$

$5^{\prime}-O$-(4,4'-Dimethoxytrityl)-2'-deoxythymidine (5'-O-DMTr-T)

Argon (or, optionally, nitrogen), dry

Acetonitrile, anhydrous

2-Chloro-spiro-4,4-pentamethylene-1,3,2-oxathiaphospholane (S.9 or labeled

S.12; see Basic Protocol 1 or Alternate Protocol 1)

Elemental sulfur, anhydrous (dried overnight at high vacuum)

Chloroform (distilled with $1 \mathrm{~mL}$ pyridine per $\mathrm{L}$ )

Toluene, dry

Pyridine, anhydrous

Diisopropylethylamine (Aldrich), anhydrous

Diphenylcarbamoyl chloride (Aldrich)

9:1 (v/v) chloroform/methanol

Merck $60 \mathrm{H}$ silica gel, particle size 5 to $40 \mu \mathrm{m}$

Ethyl acetate

Butyl acetate

Benzene

25-mL two-neck round-bottom flasks

High-vacuum valve (Rotaflo, Quickfit)

Rubber septum

High-vacuum oil pump $(0.01 \mathrm{mmHg})$

2-mL and 10-mL gas-tight syringes

Buchner funnel

$25 \times 3-\mathrm{cm}$ chromatography column packed with $20 \mathrm{~g}$ of 230 to 400 mesh silica gel

TLC silica gel plates with UV indicator (Merck; also see APPENDIX 3D)

Constant temperature water bath

$30 \times 2-\mathrm{cm}$ chromatography column

Filter paper (Whatman no. 1)

High-performance TLC (HP-TLC) plates (silica gel $60 \mathrm{~F}_{254}$ ) with UV indicator

(Merck; also see APPENDIX 3D)

Additional reagents and equipment for column chromatography (APPENDIX $3 E$ ) and thin-layer chromatography (TLC; APPENDIX 3D)

\section{Phosphitylate protected deoxyribonucleosides}

1. Place $10 \mathrm{mmol}$ of $5^{\prime}$-O-DMTr- $N$-protected deoxyribonucleoside $\left(\mathrm{dA}^{\mathrm{Bz}}, \mathrm{dG}^{i-\mathrm{Bu}}, \mathrm{T}\right.$, or $\mathrm{dC}^{\mathrm{Bz}}$ ) in a $25-\mathrm{mL}$ two-neck round-bottom flask containing a magnetic stir bar, with a high-vacuum valve in one joint and a rubber septum in the other, and dry overnight at high vacuum $(0.01 \mathrm{mmHg}$; provided by high-vacuum oil pump).

2. Close the vacuum valve and fill the flask with dry argon (optionally nitrogen).

3. Using a gas-tight syringe, add $10 \mathrm{~mL}$ of anhydrous acetonitrile through the septum.

4. Using a gas-tight syringe, add $1.91 \mathrm{~mL}(11 \mathrm{mmol})$ of anhydrous diisopropylethylamine.

Synthesis of Modified Oligonucleotides and Conjugates

4.17.13

Supplement 15 
5. To the magnetically stirred solution, using a gas-tight syringe, add dropwise at room temperature $1.63 \mathrm{~mL}(2.32 \mathrm{~g}, 11 \mathrm{mmol})$ of 2-chloro-spiro-4,4-pentamethylene-1,3,2oxathiaphospholane (S.9) over a 5-min period.

6. Stir an additional $5 \mathrm{~min}$ and add $\sim 0.5 \mathrm{~g}(\sim 15 \mathrm{mmol})$ dry elemental sulfur. Continue stirring for $12 \mathrm{hr}$.

7. Filter off excess sulfur using a Buchner funnel. Evaporate the solvent and dissolve the residue in $4 \mathrm{~mL}$ of chloroform (distilled with pyridine).

8. Apply crude product to a $25 \times 3-\mathrm{cm}$ column packed with $20 \mathrm{~g}$ of 230 to 400 mesh silica gel. Elute the column with chloroform, collecting 8- to $10-\mathrm{mL}$ fractions. Identify the appropriate fractions by TLC (APPENDIX $3 D$ ) using standard silica gel plates and 95:5 (v/v) $\mathrm{CHCl}_{3} /$ methanol $\left(R_{\mathrm{f}}=0.6575\right)$. Combine fractions and evaporate the solvents under reduced pressure ( 15 to $20 \mathrm{mmHg}$ ) with a water bath temperature not exceeding $30^{\circ} \mathrm{C}$.

9. Add 5 to $6 \mathrm{~mL}$ of dry toluene and evaporate to dryness with exclusion of moisture using a membrane pump. Repeat this step twice.

After the solvent is evaporated, apply high vacuum to the flask for $2 \mathrm{hr}$. Close the flask with a septum and pierce the septum with a needle. Store the flask in a desiccator and apply high vacuum for $\geq 12 \mathrm{hr}$. The monomers can be stored at room temperature in a desiccator for a month.

The desired 5'-O-DMTr-deoxyribonucleoside-3'-O-(2-thio-spiro-4,4-pentamethylene1,3,2-oxathiaphospholane)s are obtained in $75 \%$ to $85 \%$ yield. For the guanosine derivative, diphenylcarbamoyl protection at $\mathrm{O6}$ is required (steps 10 to 15). The diastereomeric composition, ${ }^{31} P$ NMR chemical shifts, and TLC parameters (HP-TLC plates) of compounds S.3a to $\boldsymbol{c}$ are given in Table 4.17.1. Elemental analysis (found/calculated): $\boldsymbol{S} . \mathbf{3 a}$ (B $=$ T) $C 61.67 \% / 60.79 \%, H 6.15 \% / 5.77 \%, N 3.73 \% / 3.73 \%, P 4.06 \% / 4.13 \%, S$ 8.00\%/8.54\%; S.3b $\left(B=A^{B z}\right) C 62.84 \% / 62.56 \%$, H $5.48 \% / 5.37 \%, N$ 7.73\%/8.11\%, $P$ $3.41 \% / 3.58 \%$, S 6.74\%/7.42\%; S.3c $\left(B=C^{B z}\right) C 62.53 \% / 62.92 \%, H 5.47 \% / 5.52 \%, N$ $5.14 \% / 5.00 \%, P 3.65 \% / 3.69 \%, S 7.26 \% / 7.63 \%$.

Table 4.17.1 Characteristics of the 5'-O-DMTr-deoxyribonucleoside-3'-O-(2-thio-spiro-4,4pentamethylene-1,3,2-oxathiaphospholane) $s^{a}$

\begin{tabular}{|c|c|c|c|c|}
\hline Base & Yield (\%) & $\begin{array}{c}\text { Composition } \\
\text { (fast:slow) }\end{array}$ & $\begin{array}{c}\delta_{\mathrm{P}} \\
\left(\mathrm{ppm}, \mathrm{CD}_{3} \mathrm{CN}\right)\end{array}$ & $R_{\mathrm{f}}(\mathrm{TLC})^{b}$ \\
\hline $\mathrm{T}(\mathbf{3 a})$ & 84 & $50: 50$ & $\begin{array}{l}105.3 \text { (fast) } \\
105.6 \text { (slow) }\end{array}$ & $\begin{array}{c}0.61 \text { (fast) } \\
0.54 \text { (slow) }\end{array}$ \\
\hline $\mathrm{A}^{\mathrm{Bz}}(\mathbf{3 b})$ & 84 & $49: 51$ & $\begin{array}{l}104.7 \text { (fast) } \\
105.1 \text { (slow) }\end{array}$ & $\begin{array}{c}0.54 \text { (fast) } \\
0.46 \text { (slow) }\end{array}$ \\
\hline $\mathrm{C}^{\mathrm{Bz}}(\mathbf{3 c})$ & 86 & $48: 52$ & $\begin{array}{l}105.3 \text { (fast) } \\
105.6 \text { (slow) }\end{array}$ & $\begin{array}{c}0.60 \text { (fast) } \\
0.40 \text { (slow) }\end{array}$ \\
\hline $\mathrm{G}^{i-\mathrm{Bu}, \mathrm{DPC}}(\mathbf{3 d})$ & 78 & $52: 48$ & $\begin{array}{c}106.2{\text { (fast })^{d}} \\
106.9 \text { (slow) }^{d}\end{array}$ & $\begin{array}{l}0.37 \text { (fast }^{c} \\
0.26 \text { (slow) }^{c}\end{array}$ \\
\hline
\end{tabular}

${ }^{a}$ Table adapted from Stec et al. (1998) with permission from the American Chemical Society.

${ }^{b}$ TLC performed on HP-TLC plates with UV indicator (Merck) and a developing system of 1:1 (v/v) butyl acetate/benzene $\left(\mathrm{T}\right.$ and $\left.\mathrm{G}^{i-\mathrm{Bu}}\right)$ or 1:2 (v/v) ethyl acetate/butyl acetate $\left(\mathrm{A}^{\mathrm{Bz}}\right.$ and $\left.\mathrm{C}^{\mathrm{Bz}}\right)$.

Synthesis of Phosphorothioate Oligonucleotides with Stereodefined Linkages

\subsubsection{4}

${ }^{c} R_{\mathrm{f}}$ values reported for monomers before $O^{6}$-protection with diphenylcarbamoyl chloride (DPC). After protection, $R_{\mathrm{f}}$ values are 0.74 and 0.63 using the same solvent system.

${ }^{d} \delta_{\mathrm{P}}$ values reported for monomers after $O^{6}$-protection with DPC. 
10. Place $0.85 \mathrm{~g}(1 \mathrm{mmol})$ of $N^{2}$-isobutyryl-5'-O-(4,4'-dimethoxytrityl)-3'-O-(2-thiospiro-4,4-pentamethylene-1,3,2-oxathiaphospholanyl)-2'-deoxyguanosine (from step 9) in a two-neck $25-\mathrm{mL}$ round-bottom flask with a magnetic stir bar, with a high-vacuum valve in one neck and a rubber septum in the other, and dry overnight at high vacuum $(0.01 \mathrm{mmHg})$.

11. Using a $10-\mathrm{mL}$ gas-tight syringe, add $5 \mathrm{~mL}$ of anhydrous pyridine.

12. Using a $2-\mathrm{mL}$ gas-tight syringe, add $0.26 \mathrm{~mL}(1.5 \mathrm{mmol})$ diisopropylethylamine and $0.46 \mathrm{~g}(2.0 \mathrm{mmol})$ diphenylcarbamoyl chloride, with stirring, at room temperature. Continue stirring mixture $1 \mathrm{hr}$.

13. Concentrate mixture to dryness, dissolve in $1.5 \mathrm{~mL}$ of chloroform, and apply on a $25 \times 3-\mathrm{cm}$ column packed with $20 \mathrm{~g}$ of 230 to 400 mesh silica gel. Elute the column with $300 \mathrm{~mL}$ chloroform, collecting 10 - to $12-\mathrm{mL}$ fractions.

14. Analyze fractions by TLC (APPENDIX 3D) on standard silica gel plates. Develop TLC plates with 9:1 (v/v) chloroform/methanol.

15. Combine all fractions that contain the desired product $\left(R_{\mathrm{f}}=0.79\right)$. Evaporate the solvents under reduced pressure ( 15 to $20 \mathrm{mmHg}$ ) with the temperature of the water bath not exceeding $35^{\circ} \mathrm{C}$. Dissolve the residue in dry toluene and evaporate the solvent. Store the pure product $\mathbf{S . 3 d}$ (a pale yellow oil) in a tightly closed vessel.

Approximately $0.95 \mathrm{~g}(90 \%$ to $95 \%$ yield $)$ should be isolated. $\mathrm{MS} .(+\mathrm{FAB}) \mathrm{m} / \mathrm{z} 1041.6\left(\mathrm{M}^{+}\right.$, $1 \%), \mathrm{m} / \mathrm{z}, 1042.6\left(\mathrm{M}^{+}+1,0.6 \%\right), \mathrm{m} / \mathrm{z} 303.2\left(\mathrm{DMTr}^{+}, 100 \%\right)$. Elemental analysis(found/calculated): C 63.60\%/63.44\%, H 5.78\%/5.52\%, N 7.77\%/8.08\%, P 2.72\%/2.98\%, S $5.78 \% / 6.15 \%$. The diastereomeric composition, ${ }^{31} \mathrm{P}$ NMR chemical shifts, and TLC parameters (HP-TLC plates) of $\mathbf{S . 3 d}$ are given in Table 4.17.1.

\section{Separate diastereomers of S.3a to d}

16. The day before separation, load a $30 \times 2-\mathrm{cm}$ column with a degassed suspension of $\sim 20 \mathrm{~g}$ of silica gel (Merck $60 \mathrm{H}$, particle size 5 to $40 \mu \mathrm{m}$ ) in $\sim 100 \mathrm{~mL}$ of the appropriate mixture of solvents:

2:1:0.003 (v/v/v) ethyl acetate/butyl acetate/pyridine for $\mathrm{dA}$ and $\mathrm{dC}$ derivatives

1:2:0.003 (v/v/v) ethyl acetate/butyl acetate/pyridine for $\mathrm{dG}$ derivative 1:1:0.002 (v/v/v) butyl acetate/benzene/pyridine for T monomer.

Gently cover the top surface of the gel with a disc of Whatman no.1 filter paper of diameter close to the inside diameter of the column. Flush the column with $150 \mathrm{~mL}$ of the eluant. Maintain a 2- to 3-mm layer of eluant over the gel.

IMPORTANT NOTE: Because the differences in chromatographic mobilities of P-diastereomers are very small, the glass frit must be mounted within the cylindrical part of the column to assure undisturbed, laminar flow of the eluant. To achieve good resolution, the column should be packed with the silica gel suspension at least $24 \mathrm{hr}$ before chromatography. Since isocratic elution is used for the separation, the same column may be used for two to three consecutive separations of a given monomer.

17. Dissolve $\sim 300 \mathrm{mg}$ of a monomer (mixture of diastereomers) in $1.5 \mathrm{~mL}$ of the appropriate eluant (see step 16) and apply gently on the gel.

18. Elute the column with $300 \mathrm{~mL}$ of appropriate eluant and collect $10-$ to $12-\mathrm{mL}$ fractions. Analyze fractions by TLC on HP-TLC plates (Table 4.17.1). Combine appropriate fractions and concentrate to dryness under reduced pressure (15 to 20 $\mathrm{mmHg}$ ) with the temperature of the water bath not exceeding $35^{\circ} \mathrm{C}$.

Synthesis of Modified Oligonucleotides and Conjugates

4.17.15

Supplement 15 
ALTERNATE PROTOCOL 2

Synthesis of Phosphorothioate Oligonucleotides with Stereodefined Linkages

4.17.16
19. Evaporate the pure diastereomers twice with anhydrous toluene, with exclusion of moisture, and store in a tightly closed vessel for up to 1 month at room temperature.

Typically, for $d A, d T$, and $d C$ monomers, one passage gives $75 \%$ to $80 \%$ separated diastereomers of $96 \%$ to $100 \%$ diastereomeric purity, as assessed by ${ }^{31} P$ NMR. For the $d G$ derivative, the "fast" isomer is usually obtained in lower yield (28\% to 30\%, $100 \%$ diastereomeric purity) while the "slow" isomer is obtained in $50 \%$ yield, but only of $90 \%$ diastereomeric purity, and must be rechromatographed.

\section{SYNTHESIS OF 5'-O-DMTr-DEOXYRIBONUCLEOSIDE-3'-O-(2-OXO-spiro- 4,4-PENTAMETHYLENE-1,3,2-OXATHIAPHOSPHOLANE)S}

5'-O-DMTr-deoxyribonucleoside-3'-O-(2-oxo-spiro-4,4-pentamethylene-1,3,2-oxathiaphospholane)s (S.4) are synthesized from their corresponding 2-thio monomers (S.3; see Basic Protocol 2) and can be used to elongate PS-oligos obtained via the oxathiaphospholane approach and thereby generate short unmodified oligonucleotide segments. This goal cannot be achieved using standard phosphoramidite chemistry because, during the routine oxidation step by means of $\mathrm{I}_{2} /$ water/pyridine, the diester phosphorothioate linkages already present in the oligomer would undergo PS-to-PO conversion. The oligonucleotide synthesis protocol differs from that used for the synthesis of PS-oligos in the following ways: (1) because of their relatively low stability, the 2-oxo-monomers should not be repurified before synthesis (see Basic Protocol 3, step 1); and (2) the amount of DBU necessary for condensation (step 16) may be reduced by $50 \%$.

\section{Materials}

5'-O-DMTr-deoxyribonucleoside-3'-O-(2-thio-spiro-4,4-pentamethylene-1,3,2oxathiaphospholane)s (S.3; see Basic Protocol 2)

Silica gel 60, 230 to 400 mesh

Acetonitrile, anhydrous

Argon (or, optionally, nitrogen), dry

Selenium dioxide, anhydrous (dried overnight at high vacuum)

95:5 (v/v) chloroform/methanol (distill chloroform with $1 \mathrm{~mL}$ pyridine per $\mathrm{L}$ )

Two-neck 10-mL round-bottom flask

High-vacuum valve (e.g., Rotaflo, Quickfit)

Rubber septum

High-vacuum oil pump

$10-\mu$ mol-scale DNA synthesis column

1- to 2-mL polypropylene syringes with luer ends

5-mL gas-tight syringe

TLC silica gel plates with UV indicator (Merck; also see APPENDIX $3 D$ )

10-mL gas-tight syringe

Luer male-to-male adapter

Additional reagents and equipment for thin-layer chromatography (TLC; APPENDIX 3D)

CAUTION: Selenium dioxide is toxic.

1. Place $\sim 300 \mathrm{mg}$ of $5^{\prime}$ - $O$-DMTr-deoxyribonucleoside-3'-O-(2-thio-spiro-4,4-pentamethylene-1,3,2-oxathiaphospholane) $(\mathbf{S . 3})$ in a two-neck $10-\mathrm{mL}$ round-bottom flask containing a magnetic stir bar, with a high-vacuum valve in one joint and a rubber septum in the other, and dry overnight at high vacuum $(0.01 \mathrm{mmHg}$; provided by high-vacuum oil pump). 
2. Assemble a 10- $\mu$ mol-scale DNA synthesis column filled to $80 \%$ of its volume with silica gel 60 (230 to 400 mesh). Wash the gel three times, each time with $5 \mathrm{~mL}$ anhydrous acetonitrile. Apply a stream of dry argon and continue drying for 15 to 20 min after the gel loosens. Close both ends of the column with two 1- to 2-mL luer syringes to eliminate contact with atmospheric moisture.

The dryness of the gel in the column is crucial for stability of the product. Every effort should be made to protect the dried gel from moisture.

3. Close the vacuum valve on the flask containing dried $\mathbf{S . 3}$ and fill the flask with dry argon.

4. Using a 5-mL gas-tight syringe, add $4 \mathrm{~mL}$ of anhydrous acetonitrile.

5. With continuous flow of dry argon, remove the vacuum valve and add, in several portions, $\sim 80 \mathrm{mg}$ selenium dioxide.

6. Monitor the progress of the reaction by TLC (APPENDIX $3 D)$ on silica gel plates. Develop TLC plates with 95:5 (v/v) chloroform/methanol (see Table 4.17.2 for $R_{\mathrm{f}}$ values of S.4).

Because the differences in chromatographic mobilities are small, always apply the starting material on an adjacent lane as a reference.

7. After the reaction is complete, withdraw the supernatant with a 10 -mL gas-tight syringe and remove the needle.

8. Attach a luer male-to-male adapter to the bottom of the silica gel column and gently load the withdrawn supernatant at the top. Collect the effluent in a dry flask with continuous flow of dry argon. Wash the silica gel with 1 to $2 \mathrm{~mL}$ of dry acetonitrile.

As filtration progresses, an orange layer of selenium compounds moves through the gel to the bottom of the column. Stop the filtration when the layer reaches two-thirds of the column length from the top.

9. Replace the high-vacuum valve on the flask and evaporate acetonitrile at high vacuum. Apply the vacuum slowly while shaking the flask continuously until the oil residue makes a foam.

5'-O-DMTr-deoxyribonucleoside-3'-O-(2-oxo-spiro-4,4-pentamethylene-1,3,2-oxathiaphospholane)s (S.4) are unstable and should be used for synthesis within a few hours after preparation. They are typically isolated at $40 \%$ to $55 \%$ yield. Their ${ }^{31} \mathrm{P} N \mathrm{NR}$ chemical shifts and TLC parameters are reported in Table 4.17.2.

Table 4.17.2 Characteristics of the 5'-O-DMTr-deoxyribonucleoside-3'-O-(2-oxo-spiro-4,4pentamethylene-1,3,2-oxathiaphospholane) $s^{a}$

\begin{tabular}{lccc}
\hline Base & Yield $(\%)$ & $\begin{array}{c}\delta_{\mathrm{P}} \\
\left(\mathrm{ppm}, \mathrm{CD}_{3} \mathrm{CN}\right)\end{array}$ & $R_{\mathrm{f}}(\mathrm{TLC})^{b}$ \\
\hline $\mathrm{T}(\mathbf{4 a})$ & 55 & $44.7,44.3$ & 0.71 \\
$\mathrm{~A}^{\mathrm{Bz}}(\mathbf{4 b})$ & 41 & $45.1,44.9$ & 0.71 \\
$\mathrm{C}^{\mathrm{Bz}}(\mathbf{4 c})$ & 45 & $44.6,44.1$ & 0.70 \\
$\mathrm{G}^{i-\mathrm{Bu}, \mathrm{DPC}}(\mathbf{4 d})$ & 54 & $45.3,44.5$ & 0.74 \\
\hline
\end{tabular}

${ }^{a}$ Table adapted from Stec et al. (1998) with permission from the American Chemical Society.

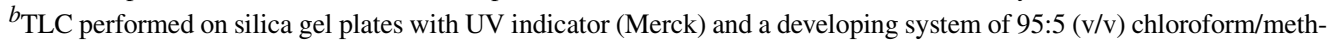
anol. The 2-oxo-monomers migrate slightly more slowly than their 2-thio-precursors.

Synthesis of Modified Oligonucleotides and Conjugates

4.17.17

Supplement 15 


\section{MANUAL SOLID-PHASE SYNTHESIS OF STEREODEFINED OLIGO(NUCLEOSIDE PHOSPHOROTHIOATE)S}

Synthesis of oligo(nucleoside phosphorothioate)s using the oxathiaphospholane method can be performed either in a standard $1-\mu \mathrm{mol}$ column $(1-\mu \mathrm{mol}$-scale synthesis) or in a reassembled OPC column (2- $\mu \mathrm{mol}$ scale; see below). Because of the strong base used for the condensation step (1,4-diazabicyclo[5.4.0] undec-7-ene), a sarcosinylated solid support must be used (see Support Protocol 2). Typically, supports are functionalized with 25 to $40 \mu \mathrm{mol}$ nucleoside per gram. A lower nucleoside concentration is not recommended, because a larger amount of support will be necessary to achieve the synthesis at the recommended scale, and may not leave enough space for efficient mixing with incoming reagents. The condensation step is extremely sensitive even to trace amounts of moisture. Therefore, the acetonitrile to be used as a solvent for DBU, and deoxyribonucleoside oxathiaphospholane monomers should be dried over $\mathrm{P}_{2} \mathrm{O}_{5}(5 \mathrm{~g} / \mathrm{L})$ and distilled under reduced pressure $(\sim 200 \mathrm{mmHg})$ through a 20 -cm Vigreux column with exclusion of moisture under an atmosphere of dry argon. At least one-third of the initial volume must remain in the flask. Acetonitrile dried in this way must be transferred using a gas-tight syringe under an atmosphere of dry argon, or by the vacuum line technique (see Support Protocol 1).

This protocol describes a $2-\mu$ mol-scale synthesis. The synthesis proceeds from the 3 '-end to the $5^{\prime}$-end of the sequence. The first nucleoside from the $3^{\prime}$-end is attached to the solid support. To avoid mistakes, the investigator is recommended to have a synthesis step check list to check off each of the executed steps during consecutive synthetic cycles, as illustrated in Table 4.17.3 for synthesis of the sequence $5^{\prime}$-TGACTGCA-3'. Notably, after the last condensation step, the capping procedure is not executed.

\section{Materials}

Deoxyribonucleoside oxathiaphospholane monomers (see Basic Protocol 2 and/or Alternate Protocol 2)

Chloroform (optional), distilled with $1 \mathrm{~mL}$ pyridine per $\mathrm{L}$

Toluene, anhydrous

Low-pressure argon or nitrogen, dried (see recipe)

Sarcosinylated solid support functionalized with a nucleoside (first from the $3^{\prime}$ end of sequence to be synthesized) at a concentration ranging from 20 to $30 \mu \mathrm{mol} / \mathrm{g}$ support (see Support Protocol 2)

Capping reagent A: 1:1:8 (v/v/v) acetic anhydride/pyridine/tetrahydrofuran (THF)

Capping reagent B: $7 \mathrm{~g}$ 4-dimethylaminopyridine/93 mL THF

Anhydrous acetonitrile $\left(\mathrm{H}_{2} \mathrm{O}<20 \mathrm{ppm}\right.$; see recipe $)$ in bottle with rubber septum, with dry gas delivered inside through a line ending in a needle

Table 4.17.3 Sample Check List for Oligonucleotide Synthesis (Sequence 5'-TGACTGCA-3')

Synthesis of Phosphorothioate Oligonucleotides with Stereodefined Linkages

4.17.18 
Detritylating reagent: $3.5 \%(\mathrm{w} / \mathrm{v})$ dichloroacetic acid in methylene chloride

Acetonitrile, HPLC grade (Baker)

1:4.5 (v/v) 1,8-diazabicyclo[5.4.0]undec-7-ene (DBU) in anhydrous acetonitrile $\left(\mathrm{H}_{2} \mathrm{O}<20 \mathrm{ppm}\right)$

Methylene chloride

Aqueous ammonia, concentrated (Baker)

$10 \times 3-\mathrm{cm}$ chromatography column packed with $10 \mathrm{~g} 230$ - to 400-mesh silica gel 60 (optional)

4-mL sample vials with open-top screw caps with Teflon-faced rubber septa

19- to 22-G, 1- to 1.5-in. luer-lock needles

Vacuum desiccator $(\leq 0.05 \mathrm{mmHg}$; provided by high-vacuum oil pump) with condenser cooled by liquid $\mathrm{N}_{2}$

1-, 2-, and 5-mL all-polypropylene luer-lock syringes

19- to 22-G, 2- to 3-in. luer-lock needles with blunt $90^{\circ}$ tips

Columns for DNA synthesis:

For 1- $\mu$ mol scale: Applied Biosystems DNA synthesis column (cat. no. 400407), empty, $1.0 \mu \mathrm{mol}$ crimp-style

For 2- $\mu$ mol scale: emptied and reassembled Applied Biosystems oligonucleotide purification cartridge (OPC; cat. no. 400771)

Column filters (two for each column; Applied Biosystems, cat. no. 400059)

Aluminum seals (caps; two for each column; Aldrich cat. no. Z11413-8; Wheaton aluminum cap, $13 \mathrm{~mm}$, tear-off)

Crimper for aluminum seals (Aldrich, cat. no. z 11423)

Polypropylene (or other chemically inert) luer male-to-male adapter

100- and 500- $\mu \mathrm{L}$ gas-tight syringes (Hamilton)

Glass drying tube ( 2-cm i.d., 25-cm length), with three-way valve (or two independent valves) at the top and a rubber septum at the opposite side

High-vacuum $(0.05 \mathrm{mmHg})$ oil pump

500-mL filtering flask capped with a rubber septum pierced with a 3- to 4-mm i.d. hole

Water aspirator with a manostat

Two waste bottles: one for chlorinated waste (methylene chloride, detritylating reagent) and another for water-miscible wastes (acetonitrile, capping reagents $\mathrm{A}$ and $\mathrm{B}$ )

$60^{\circ} \mathrm{C}$ water bath (optional)

Speedvac concentrator with vacuum provided by water aspirator

19- to 22-G, 4- to 5-in. luer-lock needles with blunt $90^{\circ}$ tips

Additional reagents and equipment for column chromatography (optional; APPENDIX 3E) and purification of oligonucleotides (UNITS 10.3-10.5)

CAUTION: The drying tube must be tested to make sure that it is safe for high-vacuum usage, i.e., at a pressure lower than $0.05 \mathrm{mmHg}$.

\section{Prepare monomers for synthesis}

1. If the deoxyribonucleoside oxathiaphospholane monomers ( $\mathbf{S} . \mathbf{3}$ only) have been stored for more than one week, repurify them by flash chromatography (APPENDIX $3 E$ ) on a $10 \times 3-\mathrm{cm}$ silica gel column using chloroform (distilled with pyridine) as eluant. Concentrate appropriate fractions with exclusion of moisture. Coevaporate the residue twice with anhydrous toluene and apply high vacuum to generate a foam.

Repurification is necessary to keep repetitive yield at $92 \%$ to $94 \%$. The 2-oxo-monomers (S.4) should not be repurified in this manner, and should be used within a few hours of synthesis (see Alternate Protocol 2).

Synthesis of Modified Oligonucleotides and Conjugates

4.17.19 
2. If necessary, use a spatula to disaggregate the foam of oxathiaphospholane monomers.

3. Put $\sim 30 \mathrm{mg}$ of given monomer (S.3 or $\mathbf{S . 4}$ ) in an appropriately marked 4-mL sample vial (one vial for each condensation step to be performed) and cover tightly with an open-top screw cap with a Teflon-faced rubber septum. Pierce each septum with a 19- to 22-G, 1- to 1.5-in. luer-lock needle.

Before the vials are stored in the desiccator (step 4), be sure that none of the needles became clogged during piercing. This can be done by temporarily inserting a second needle to deliver a stream of dry argon to the vial. The unrestricted flow of argon from the first needle should be easily sensed.

4. Dry monomers in a vacuum desiccator at $\leq 0.05 \mathrm{mmHg}$ for at least $12 \mathrm{hr}$. After the drying is complete, fill the desiccator with dry argon, open it cautiously, and immediately remove the needles from the vials.

The condenser should be free of any solvents before the drying starts. It is recommended that the condenser be cooled with liquid nitrogen. It is imperative that the oil pump and the whole vacuum system intended for drying monomers be free of acidic impurities such as acetic or hydrochloric acid. Traces of acids would partially detritylate the monomers.

\section{Perform initial setup procedures}

5. Prepare and place a permanent mark upon all-polypropylene syringes for capping (2 $\mathrm{mL})$, detritylation $(5 \mathrm{~mL})$, washing with acetonitrile $(5 \mathrm{~mL})$, and washing with methylene chloride ( $5 \mathrm{~mL}$ ). Fit each with a 19- to $22-\mathrm{G}, 2$ - to 3 -in. needle with a blunt $90^{\circ}$ tip.

Use the syringes only for designated purposes, as cross-contamination may lead to poor synthesis yields. The needles should be blunted for safety reasons.

6. Fill a column with an appropriate support for synthesis and assemble it. Be sure to insert column filters at both ends of the column. Secure the aluminum caps with a crimper. Remove the middle part of each aluminum cap. Insert a luer male-to-male adapter into one outlet of the column and attach the outlet needle (19- to 22-G, 4- to 5 -in. with a blunt $90^{\circ}$ tip).

Do not hesitate to apply significant force to secure aluminum caps. This is crucial for avoiding leakage of chemicals during synthesis. The long outlet needle (4- to 5-in.) is more convenient for swirling of the column during consecutive synthetic steps.

7. Set the manostat for the filtering flask at 400 to $450 \mathrm{mmHg}$.

\section{Start synthesis cycle}

8. Mix $1 \mathrm{~mL}$ capping reagent $\mathrm{A}$ and $1 \mathrm{~mL}$ capping reagent $\mathrm{B}$ in the 2 -mL dedicated syringe for capping (see step 5), remove the needle, and gently load the mixture into the column. Continue capping for 2 min with intermittent swirling of the column. Expel reagent from the column into a proper waste bottle.

CAUTION: Avoid undue force when pushing the plunger. Excessively fast loading or expelling of the reagents may damage the column filters.

9. Fill the dedicated 5-mL syringe for acetonitrile washing (see step 5) with HPLC-grade acetonitrile. Remove the needle and flush the column with intermittent swirling. Collect the effluent in the proper waste bottle. Expel the remaining liquid, then insert the outlet needle of the column through the septum on the top of the filtering flask, deliver a stream of argon from the top of the column, and gently apply suction to the filtering flask. Continue drying until the support in the column becomes loose. 
10. Fill the dedicated 5-mL syringe for detritylation (see step 5) with detritylating reagent. Remove the needle and flush the column with intermittent swirling. Collect the effluent in the proper waste bottle or in a test tube for quantitative cationic DMTr analyses. Expel the remaining liquid.

IMPORTANT NOTE: Upon contact with the support, the detritylation solution becomes red due to the presence of DMTr cations removed from the nucleoside. If at the end of delivery the effluent is still colored, continue detritylation with an additional volume of fresh reagent to complete the detritylation process.

11. Wash the support with $5 \mathrm{~mL}$ of acetonitrile and dry it as described in step 9 .

12. Put the column and gas-tight syringes $(500 \mu \mathrm{L}$ and $100 \mu \mathrm{L})$ in the drying tube, gently apply high vacuum, and continue drying for $10 \mathrm{~min}$. Close the vacuum valve on the drying tube and deliver dry gas to the tube. Take the syringes out of the tube (leave the column inside), close the tube with the septum, close the gas valve, and apply vacuum again. Use the syringes only for designated purposes.

CAUTION: Do not allow the rubber septum to be expelled from the bottom of the tube by excessively high gas pressure.

13. Withdraw $300 \mu \mathrm{L}$ of dry acetonitrile $\left(\mathrm{H}_{2} \mathrm{O}<20 \mathrm{ppm}\right)$ using the $500-\mu \mathrm{L}$ gas-tight syringe and add the solvent to the vial containing the appropriate oxathiaphospholane monomer.

14. Close the vacuum valve on the drying tube and deliver dry gas to the tube. Take the column out of the tube, close the tube with the septum, and close the gas valve. Insert a dry 1-mL syringe into the inlet of the column.

15. Withdraw $90 \mu \mathrm{L}$ of $1: 4.5$ (v/v) DBU/acetonitrile using the $100-\mu \mathrm{L}$ gas-tight syringe and add the reagent to the vial containing the dissolved oxathiaphospholane monomer. Mix the contents of the vial for a few seconds.

16. Pierce the septum of the vial with the needle of the column and, using the syringe attached to the column, suck up the contents of the vial to fill the column. Swirl the column intermittently for $10 \mathrm{~min}$. Expel the liquid to the waste bottle.

17. Wash the support with $5 \mathrm{~mL}$ of methylene chloride, followed by $5 \mathrm{~mL}$ of HPLC-grade acetonitrile, and dry as described in step 9.

\section{Complete synthesis cycle}

18. Repeat steps 8 to 17 with each successive monomer until elongation is complete.

19. Execute a routine cleavage of the product from the support using a few milliliters of concentrated aqueous ammonia for $2 \mathrm{hr}$, followed (if necessary) by heating of the ammoniacal solution in a tightly closed vessel at $55^{\circ} \mathrm{C}$ for $12 \mathrm{hr}$ to remove nucleobase-protecting groups.

20. Concentrate the sample under reduced pressure in a Speedvac concentrator using a water aspirator.

21. Purify the oligonucleotide according to published protocols (UNITS 10.3-10.5).

Synthesis of Modified Oligonucleotides and Conjugates

4.17.21 

COSINYLATED SOLID-PHASE SUPPORT

This protocol attaches $5^{\prime}$ - $O$-DMTr- $N$-protected nucleoside- $3^{\prime}$ - $O$-succinyl hemiesters to long-chain alkylamine controlled-pore glass (LCAA-CPG) through a sarcosinyl linker. Since $5^{\prime}$ - $O$-DMTr- $N$-protected nucleoside- $3^{\prime}$ - $O$-succinate hemiesters are commercially available, their syntheses are not described. Alternatively, one may prepare them by coupling succinic anhydride with a protected nucleoside according to published procedures (see UNIT 3.2 and references therein).

\section{Materials}

Long-chain alkylamine controlled-pore glass (LCAA-CPG) beads (80 to 120 mesh, $500 \AA$ Å; Sigma)

9-Fluorenylmethoxycarbonyl (Fmoc)-sarcosine monohydrate (Fluka)

Argon (or, optionally, nitrogen), dry

1,3-Dicyclohexylcarbodiimide (DCC; Aldrich)

Dimethylformamide (DMF), anhydrous

Pyridine, anhydrous

1:1:1 (v/v/v) acetonitrile/methanol (reagent grade)/pyridine

Acetonitrile, anhydrous

$10 \%(\mathrm{v} / \mathrm{v})$ piperidine in pyridine

$5^{\prime}-O$-DMTr- $N$-protected nucleoside-3'-O-succinyl hemiester (Sigma)

25-mL two-neck round-bottom flasks

High-vacuum valve (e.g., Rotaflo, Quickfit)

Rubber septum

High-vacuum oil pump

1-mL and 10-mL gas-tight syringes

Buchner funnel with glass frit

Filter flask

Vacuum source (e.g., water aspirator)

50-mL Erlenmeyer flask with stopcock

CAUTION: 1,3-Dicyclohexylcarbodiimide can cause skin or eye irritation and allergic reactions. Use appropriate protection.

\section{Couple Fmoc-sarcosine to $L C A A-C P G$}

1. Place $2 \mathrm{~g}$ LCAA-CPG beads and $0.5 \mathrm{~g}(1.6 \mathrm{mmol})$ Fmoc-sarcosine in a $25-\mathrm{mL}$ two-neck round-bottom flask containing a magnetic stir bar, with a high-vacuum valve in one joint and a rubber septum in the other, and dry overnight at high vacuum (0.01 mmHg; provided by a high-vacuum oil pump). At the end of drying, deliver dry argon (or nitrogen) to the flask through the septum.

2. Open the flask, quickly add $0.5 \mathrm{~g}(2.4 \mathrm{mmol}) \mathrm{DCC}$, and close the flask.

3. Using gas-tight syringes, add $5 \mathrm{~mL}$ anhydrous $\mathrm{DMF}$ followed by $0.5 \mathrm{~mL}$ anhydrous pyridine.

4. Stir the mixture at room temperature for $12 \mathrm{hr}$.

5. Transfer the suspension to a fritted-glass Buchner funnel and filter off the solution.

6. Wash support on the funnel three times, each time with $50 \mathrm{~mL}$ of $1: 1: 1(\mathrm{v} / \mathrm{v} / \mathrm{v})$ acetonitrile/methanol/pyridine with gentle suction.

7. Wash with $50 \mathrm{~mL}$ acetonitrile and continue suction for 2 to $3 \mathrm{~min}$. 
8. Transfer the support to a 50-mL Erlenmeyer flask, add $20 \mathrm{~mL}$ of $10 \%$ piperidine in pyridine, and seal the flask. Shake occasionally over a 30-min period. Decant the liquid.

9. Filter and wash as in steps 5 to 7.

10. Allow the support to dry at room temperature in a fume hood.

\section{Couple nucleoside-3'-O-succinyl hemiesters to LCAA-CPG-Sar}

12. Place 2 g LCAA-CPG-Sar and $\sim 0.35 \mathrm{~g}(\sim 0.5 \mathrm{mmol}) 5^{\prime}-O$-DMTr- $N$-protected nucleoside-3'-O-succinyl hemiester in a 25 -mL two-neck round-bottom flask containing a magnetic stir bar, with a high-vacuum valve in one joint and a rubber septum in the other, and dry overnight at high vacuum $(0.01 \mathrm{mmHg}$; provided by a high-vacuum oil pump). At the end of drying, deliver dry argon (or nitrogen) to the flask through the septum.

13. Open the flask, quickly add $0.2 \mathrm{~g}(1 \mathrm{mmol}) \mathrm{DCC}$, and close the flask.

14. Using gas-tight syringes, add $10 \mathrm{~mL}$ anhydrous $\mathrm{DMF}$, followed by $0.8 \mathrm{~mL}$ anhydrous pyridine.

15. Stir the mixture at room temperature for $24 \mathrm{hr}$.

16. Filter and wash as in steps 5 to 7.

17. Allow the support to dry at room temperature in a fume hood.

Typically, using long-chain alkylamine controlled-pore glass, 80 to 120 mesh, $500 \AA$ (Sigma) a concentration of 25 to $40 \mu \mathrm{mol}$ of nucleoside per gram of support is obtained. The support should be stored in a tightly closed vessel in the dark. It is stable for several years at room temperature.

IMPORTANT NOTE: The support should be prevented from contact with acidic vapors to avoid detritylation.

\section{REAGENTS AND SOLUTIONS}

Use deionized, distilled water in all recipes and protocol steps. For common stock solutions, see APPENDIX 2A; for suppliers, see SUPPLIERS APPENDIX.

\section{Acetonitrile, anhydrous $\left(\mathrm{H}_{2} \mathrm{O}<20 \mathrm{ppm}\right)$}

Dry acetonitrile over $5 \mathrm{~g} \mathrm{P}_{2} \mathrm{O}_{5}$ per $\mathrm{L}$ for at least $24 \mathrm{hr}$, then distill under reduced pressure through a $20-\mathrm{cm}$ Vigreux column with exclusion of moisture under an atmosphere of dry argon; at least one-third of the initial volume must remain in the flask. Transfer to reaction vessels using gas-tight syringes under dry argon, or by vacuum line technique (see Support Protocol 1).

IMPORTANT NOTE: For condensation steps in Basic Protocol 3, the water content of acetonitrile must be $<20$ ppm as measured by the Karl Fischer technique. "DNA/RNA synthesis grade" acetonitrile supplied by leading manufacturers is usually not suitable for this purpose unless dried as above.

\section{Low-pressure argon or nitrogen, dried}

Dry the gas by passing it through a 0.5 - to $0.7-\mathrm{m}-$ long column (3- to 4-cm i.d.) filled with blue indicator silica gel (Aldrich cat. no. 336815), then through a similar column filled with granular molecular sieves covered with $\mathrm{P}_{2} \mathrm{O}_{5}$. Delivery of inert gas under a slight positive pressure is accomplished by attaching a source of gas via tubing to a T-shaped glass connector. One arm of the connector is attached to the inlet of the first drying column; the other arm is connected to a rubber balloon. After the balloon is inflated up to a diameter of 40 to $50 \mathrm{~cm}$, the valve on the tank regulator is closed, and the internal pressure of the balloon will be sufficient to assure appropriate flow of the gas. The balloon needs to be reinflated from time to time.

Synthesis of Modified Oligonucleotides and Conjugates

4.17.23

Supplement 14 
The outlet of the second drying column should be attached through a bubbler (partially filled with mineral oil) to a manifold with 2 to 3 delivery lines, each ending with a valve and a male luer adapter.

Typically, argon has lower water content than nitrogen, therefore the drying columns have a longer life-time if argon gas is used.

\section{COMMENTARY}

\section{Background Information}

Oligo(nucleoside phosphorothioate)s are congeners of natural oligonucleotides where one of the two nonbridging oxygens in each internucleotide phosphate is replaced by sulfur. By virtue of asymmetry at the phosphorus atom, these constitute a mixture of $P$-diastereomers, and syntheses of such molecules with predetermined $P$-chirality at each internucleotide bond are challenging. While the stereocontrolled synthesis of PS-oligos is an art, even more challenging are studies on the properties of such $P$-stereodefined congeners, mainly with respect to their interactions with other biomolecules such as natural DNA, RNA, and proteins. The affinity of PS-oligos towards DNA and RNA seems to be dictated mainly by base pairing, but is also affected, to a certain extent, by their $P$-chirality. Notably, intuitive speculations (Zon et al., 1987) or molecular mechanics calculations (Jaroszewski et al., 1992; Hartmann et al., 1999) have led to conclusions contradicting the results of melting experiments performed with heteroduplexes involving $P$-stereodefined PS-oligos (Boczkowska et al., 2002). This underscores the importance of experimental verification of such hypotheses, which is possible only through access to stereodefined PS-oligos.

Physicochemical studies demonstrated that stereoregular PS-oligos of the sequence d(CG) of opposite configurations at phosphorus differ significantly in their ability to adopt the Z-conformation in high concentrations of sodium chloride (Boczkowska et al., 2000). Preliminary studies demonstrated that PS-oligos of $R_{\mathrm{P}}$-configuration containing polyadenylate sequences are able to form unusually stable triplexes with two antiparallel complementary RNA strands. The molecular basis of this phenomenon is still unknown, but the role of sulfur in the $R_{\mathrm{p}}$ configuration is essential, as neither [all- $S_{\mathrm{P}}$ ]-PS-oligomers nor unmodified DNA oligomers are able to form corresponding triplexes of comparable stability (Stec, unpub. observ.) $P$-stereodefined (PS) oligos have been used for studying the mode of action of several bacterial and human enzymes (Koziolkiewicz et al., 1997, 2001, 2002). The observation that plasma 3'-exonucleases-a class of proteins responsible for degradation of oligonucleotides in blood-are $R_{\mathrm{P}}$-selective enzymes provided the invaluable information that this enzymatic activity, detrimental toward antisense therapeutics, can be stopped by a single $S_{\mathrm{P}}$-phosphorothioate at the $3^{\prime}$ end of PS-oligonucleotides. Besides this practical aspect, elucidation of the mechanism of the nucleolytic cleavage by 3 '-exonuclease(s) provided, for the first time, the information necessary for the classification of this family of proteins (Koziolkiewicz et al., 2002). The interactions of phosphate groups with proteins is well documented. Therefore, the presented method for stereodefined labeling of internucleotide phosphate groups with sulfur and stable oxygen isotopes opens a new avenue for mechanistic studies on DNA/protein interactions at atomic resolution.

In this unit, experimental details of the oxathiaphospholane method, developed for stereocontrolled synthesis of oligo(nucleoside phosphorothioate)s, are presented. The method is based upon the synthesis of appropriately protected nucleoside 3'-O-(2-thiono-1,3,2oxathiaphospholane)s and their separation into diastereomerically pure species. It has been demonstrated that DBU-assisted condensation of these monomers with the $5^{\prime}-\mathrm{OH}$ group of a nucleoside or growing oligonucleotide in the iterative process of chain elongation on a solid support is stereospecific. Stereopurity of [all$R_{\mathrm{P}}$ ]- and [all- $S_{\mathrm{P}}$ ]-oligonucleotides has been validated via degradation with stereoselective nucleases such as snake venom phosphodiesterase (Eckstein et al., 1979) and nuclease P1 (Eckstein et al., 1983), respectively. Additional studies have also demonstrated that episulfide, which is released from the ring-opening condensation process, does not modify growing oligonucleotides.

One has to realize that this methodology is laborious and costly, and suffers from numerous imperfections which do not allow for the preparation of long PS-oligonucleotides ( $>15-$ mers). Nonetheless, the oxathiaphospholane
Phosphorothioate

Oligonucleotides with Stereodefined Linkages 4.17.24

Supplement 14 
method has provided many stereodefined PSoligonucleotides suitable for many applications, including NMR studies (Kanehara et al., 1996; Furrer et al., 1999) and biological evaluation in cell cultures, although newcomers to the field may have found it difficult to use. It is clear to the authors of this unit that new modified oxathiaphospholane monomers are highly desirable, as they would not only allow improvement in the repetitive yield of a single condensation step, but would also provide a new and efficient method for protecting internucleotide phosphorothioate diesters against oxidation, necessary for effective combination of the oxathiaphospholane method with phosphoramidite chemistry. Such a combination may provide an effective access to so-called gap-mer or chimeric constructs (Metelev et al., 1994; Pickering et al., 1996; Maier et al., 2000) consisting of oligo(nucleoside phosphate)s and $P$-stereodefined oligo(nucleoside phosphorothioate) segments. Work on these extensions to the presented method is in progress.

Various attempts at stereocontrolled synthesis of PS-oligos undertaken by other research establishments have been reported, among them efforts directed towards stereospecific synthesis of dinucleoside $3^{\prime}, 5^{\prime}$-phosphorothioates (Jin et al., 1996; Jin and Just, 1998; Wada et al., 1998; Wang and Just, 1999; Lu and Just, 2000; Oka et al., 2002), Agrawal's work on the synthesis of nearly stereopure PS-oligos (Iyer et al., 1995, 1998), and the successful stereocontrolled synthesis of longmers reported by Beaucage's laboratory (Wilk et al., 2000). These groups utilized the ingenious phosphoramidite methodology originally developed by Beaucage and Caruthers (1981). The availability of short $P$-stereodefined PSoligos (up to pentamers) following separation of diastereomers by means of RP-HPLC should also be noted (Murakami et al., 1994; Tamura, 1998).

\section{Critical Parameters and Troubleshooting}

Chemical synthesis of the phosphitylating reagent requires some skills to prevent release of the unpleasant odor characteristic of organosulfur compounds. It is recommended that glassware be cleaned using an oxidant, like sodium hypochlorite or hydrogen peroxide, to convert sulfhydryl or disulfide-containing compounds into sulfones.

As mentioned in relevant protocols, overheating of the vessels during high-vacuum distillation is dangerous, as it may lead to sponta- neous decomposition of the material inside the vessels. One should frequently monitor the pressure in the system during distillation and keep temperature within the indicated range.

For the synthesis of final phosphitylating reagent as well as for phosphitylation of nucleosides, the dryness of solvents and glassware is extremely important, because P(III) chlorides react instantly with traces of water.

Like oligonucleotide synthesis via the phosphoramidite or $H$-phosphonate approach, the dryness of monomers, solvents, and equipment used for coupling is essential for good yields in the oxathiaphospholane approach. Here, the importance of this factor is further stressed because, even with all precautions taken, the repetitive yield of coupling is between $92 \%$ and $94 \%$, and any further decrease in coupling yields due to the presence of traces of water may render oligonucleotide synthesis impractical. It is recommended that the cationic DMTr release be quantitated after each condensation step to check if the actual repetitive yield is acceptable.

\section{Anticipated Results}

Synthesis of phosphitylating reagent, although not trivial, should furnish the consecutive products with reasonable yield $(60 \%$ to $70 \%$ ). The reagent is stable and can be stored for months. The same applies to the deoxyribonucleoside 2-thio-oxathiaphospholane monomers. They can be stored in a desiccator at room temperature for several months. Following flash chromatography, the material is recovered at $>95 \%$ yield, and can then be used for oligonucleotide synthesis. Synthesis of a 15 -mer PS-oligo at a $2-\mu \mathrm{mol}$ scale usually provides 8 to $10 \mathrm{OD}$ units of pure material. This is $\sim 5 \%$ of theoretical yield. It is important that during HPLC purification only the upper part of the oligonucleotide peak be collected ( $>40 \%$ of peak height) to obtain a more homogenous oligomer.

\section{Time Considerations}

Since neither the oxathiaphosphitylating reagent nor the oxathiaphospholane monomers are commercially available, their preparation is necessary and takes a considerable amount of time. It is reasonable to assume that the synthesis of 2-chloro-spiro-4,4-pentamethylene1,3,2-oxathiaphospholane starting from cyclohexanecarboxaldehyde can be accomplished in 10 working days, provided that the technical staff prepares, simultaneously, the necessary anhydrous solvents.
Synthesis of Modified Oligonucleotides and Conjugates

4.17.25 
Phosphitylation/sulfurization of commercially available, appropriately protected nucleosides at $10 \mathrm{mmol} \mathrm{scale,} \mathrm{as} \mathrm{well} \mathrm{as} \mathrm{prepara-}$ tion of sarcosinylated supports, require 10 additional days. Separation of nucleoside monomers into $P$-diastereomerically pure species is a very important and rather difficult step. Undoubtedly, some experience is necessary to obtain good results. It should be emphasized that proper loading of the chromatographic column, as well as very careful elution and TLC analysis of effluent, are crucial for yield and diastereomeric purity of the resolved monomers. It must also be taken into account that separation of diastereomers is a time-consuming step, as only $\sim 300 \mathrm{mg}$ of monomer can be applied on a single silica gel column. The 100 to $120 \mathrm{mg}$ of each pure diastereomer recovered from that amount is sufficient for 3 to 4 coupling steps. To avoid delay resulting from laborious chromatographic purifications, it is good practice to stockpile pure diastereomers, as they are chemically stable and can be stored for long periods of time.

Once the monomers are made, the synthesis of PS-oligos proceeds much more rapidly, and typically one oligomer can be made and purified within 4 to 5 days. Usually, during manual synthesis, one can accomplish 8 to 10 couplings daily. If the synthesis is to be continued on the next day, it should be interrupted after the capping step, followed by washing and drying of the support. The synthetic column should then be stored in a desiccator.

\section{Literature Cited}

Beaucage, S.L. and Caruthers, M.H. 1981. Deoxynucleoside phosphoramidites: A new class of key intermediates for deoxypolynucleotide synthesis. Tetrahedron Lett. 22:1859-1862.

Boczkowska, M., Guga, P., Karwowski, B., and Maciaszek, A. 2000. Effect of P-chirality of internucleotide bonds on $\mathrm{B}-\mathrm{Z}$ conversion of stereodefined self-complementary phosphorothioate oligonucleotides of [PS]-d(CG) 4 and [PS]-d(GC)4 series. Biochemistry 39:1105711064.

Boczkowska, M., Guga, P., and Stec, W.J. 2002. Stereodefined phosphorothioate analogues of DNA: Relative thermodynamic stability of model PS-DNA/DNA and PS-DNA/RNA complexes. Biochemistry 41:12483-12487.

Brown, T., Pritchard, C.E., Turner, G., and Salisbury, S.A. 1989. A new base-stable linker for solidphase oligonucleotide synthesis. J. Chem. Soc. Chem. Commun. 891-893.
Eckstein, F. 2000. Phosphorothioate oligodeoxynucleotides: What is their origin and what is unique about them? Antisense Nucleic Acid Drug Dev. 10:117-121.

Eckstein, F., Burgers, P.M.J., Sathyanarayana, B.K., and Saenger, W. 1979. Crystal and molecular structure of adenosine $5^{\prime}-O$-phosphorothioate $O$ P-nitrophenyl ester (Sp diastereomer). Substrate stereospecificity of snake venom phosphodiesterase. Eur. J. Biochem. 100:585-591.

Eckstein, F., Potter, B.V.L., and Connolly, B. 1983. Synthesis and configurational analysis of a dinucleoside phosphate isotopically chiral at phosphorus. Stereochemical course of Penicillium citrum nuclease P1 reaction. Biochemistry 22:1369-1377.

Furrer, P., Billeci, T.M., Donati, A., Kojima, C., Karwowski, B., Sierzchala, A., Stec, W.J., and James, T.L. 1999. Structural effect of complete [RP]-phosphorothioate and phosphorodithioate substitution in the DNA strand of a model antisense inhibitor-target RNA complex. J. Mol. Biol. 285:1609-1621.

Guga, P., Domanski, K., and Stec, W.J. 2001. Oxathiaphospholane approach to the synthesis of P-chiral, isotopomeric deoxy(ribonucleoside phosphorothioate)s and phosphates labeled with oxygen isotope. Angew. Chem. Int. Ed. Engl. 40:610-613.

Hacia, J.G.,Wold, B.J., and Dervan, P.B. 1994. Phosphorothioate oligonucleotide-directed triple helix formation. Biochemistry 33:5367-5369.

Hartmann, B., Bertrand, H.-O., and Fermandjian, S. 1999. Sequence effects on energetic and structural properties of phosphorothioate DNA: A molecular modelling study. Nucl. Acids Res. 27:3342-3347.

Iyer, R.P., Yu, D., Ho, N.-H., Tan, W., and Agrawal, S. 1995. A novel nucleoside phosphoramidite synthon derived from 1R,2S-ephedrine. Tetrahedron: Asymmetry 6:1051-1054.

Iyer, R.P., Guo, M.J., Yu, D., and Agrawal, S. 1998. Solid-phase stereoselective synthesis of oligonucleoside phosphorothioates: The nucleoside bicyclic oxazaphospholidines as novel synthons. Tetrahedron Lett. 39:2491-2494.

Jaroszewski, J.W., Syi, J.-L., Maizel, J., and Cohen, J.S. 1992. Towards rational design of antisense DNA: Molecular modeling of phosphorothioate DNA analogues. Anticancer Drug Des. 7:253262.

Jin, Y. and Just, G. 1998. Stereoselective synthesis of dithymidine phosphorothioates using xylose derivatives as chiral auxiliaries. J. Org. Chem. 63:3647-3654.

Jin, Y., Biancotto, G., and Just, G. 1996. A stereoselective synthesis of dinucleotide phosphorothioates using chiral phosphoramidites as intermediates. Tetrahedron Lett. 37:973-976.
Synthesis of Phosphorothioate Oligonucleotides with Stereodefined Linkages 4.17.26 
Kanehara, H., Wada, T., Mizuguachi, M., and Makino, K. 1996. Influence of a thiophosphate linkage on the duplex stability: Does Sp configuration always lead to higher stability than Rp? Nucleosides Nucleotides 15:1169-1178.

Koziolkiewicz, M., Wójcik, M., Kobylanska, A., Karwowski, B., Rebowska, B., Guga, P., and Stec, W.J. 1997. Stability of stereoregular oligo(nucleoside phosphorothioate)s in human plasma: Diastereoselectivity of plasma $3^{\prime}$-exonuclease. Antisense Nucleic Acids Drug Dev. 7:43-48.

Koziolkiewicz, M., Owczarek, A., Domanski, K., Nowak, M., Guga, P., and Stec, W.J. 2001. Stereochemistry of cleavage of internucleotide bond by Serratia marcescens endonuclease. Bioorg. Med. Chem. 9:2403-2409.

Koziolkiewicz, M., Owczarek, A., Wójcik, M., Domanski, K., Guga, P., and Stec, W.J. 2002. Retention of the configuration in the action of human plasma $3^{\prime}$-exonuclease on oligo(deoxynucleoside phosphorothioate): A new method for assignment of absolute configuration at phosphorus in isotopomeric deoxyadenosine $5^{\prime}-O$ $\left[{ }^{18} \mathrm{O}\right]$ phosphorothioate. J. Am. Chem. Soc. 124:4623-4627.

Lackey, D.B. and Patel, J. 1997. Biochemical synthesis of chirally pure $R_{\mathrm{P}}$ oligonucleotide phosphorothioates. Biotechnol. Lett. 19:475-478.

Lu, Y. and Just, G. 2000. Stereoselective synthesis of RP- and SP-dithymidine phosphorothioates via chiral indolooxazaphosphorine intermediates derived from tryptophan. Angew. Chem. Int. Ed. Engl. 39:4521-4524.

Maier, M.A., Guzaev, A.P., and Manoharan, M. 2000. Synthesis of chimeric oligonucleotides containing phosphodiester, phosphorothioate, and phosphoramidate linkages. Org. Lett. 2:1819-1822.

Manoharan, M. 1999. 2'-Carbohydrate modifications in antisense oligonucleotide therapy: Importance of conformation, configuration and conjugation. Biochim. Biophys. Acta 1489:117130.

Martynov, I.V., Kruglyak, Y.L., Leibovskaya, G.A., Khromova, Z.I., and Stukov, O.G. 1969. Phosphonylated oximes: IV. Reactions of 1,3,2-dioxa- and 1,3,2-oxathiaphospholanes with $\alpha$ halogen nitroso alkanes. Zh. Obsch. Khim 39:996.

Metelev, V., Lisziewicz, J., and Agrawal, S. 1994. Study of antisense oligonucleotide phosphorothioates containing segments of oligodeoxynucleotides and $2^{\prime}$-O-methyloligoribonucleotides. Bioorg. Med. Chem. Lett. 4:29292934.

Murakami, A., Tamura, Y., Wada, H., and Makino, K. 1994. Separation and characterization of diastereoisomers of antisense oligodeoxyribonucleoside phosphorothioates. Anal. Biochem. 233:285-290.
Oka, N., Wada, T., and Saigo, K. 2002. Diastereocontrolled synthesis of dinucleoside phosphorothioates using a novel class of activators, dialkyl(cyanomethyl)ammonium tetrafluoroborates. J. Am. Chem. Soc. 124:4962-4963.

Pickering, J.G., Isner, J.M., Ford, C.M., Weir, L., Lazarovits, A., Rocnik, E.F., and Chow, L.H. 1996. Processing of chimeric antisense oligonucleotides by human vascular smooth muscle cells and human atherosclerotic plaque. Implications for antisense therapy of restenosis after angioplasty. Circulation 93:772-780.

Stec, W.J., Grajkowski, A., Koziolkiewicz, M., and Uznanski, B. 1991. Novel route to oligo(deoxyribonucleoside phosphorothioates): Stereocontrolled synthesis of P-chiral oligo(deoxyribonucleoside phosphorothioates). Nucl. Acids Res. 19:5883-5888.

Stec, W.J., Grajkowski, A., Karwowski, B., Kobylanska, A., Koziolkiewicz, M., Misiura, K., Okruszek, A., Wilk, A., Guga, P., and Boczkowska, M. 1995. Diastereomers of nucleoside 3'-O-(2thio-1,3,2-oxathia(selena)phospholanes): Building blocks for stereocontrolled synthesis of oligo(nucleoside phosphorothioate)s. J. Am. Chem. Soc. 117:12019-12029.

Stec, W.J., Karwowski, B., Boczkowska, M., Guga, P., Koziolkiewicz, M., Sochacki, M., Wieczorek, M., and Blaszczyk, J. 1998. Deoxyribonucleoside 3'-O-(2-thio- and 2-oxo-spiro-4,4-pentamethylene-1,3,2-oxathiaphospholane)s: Monomers for stereocontrolled synthesis of oligo(deoxyribonucleoside phosphorothioate)s and chimeric PS/PO oligonucleotides. J. Am. Chem. Soc. 120:7156-7167.

Stein, C.A. and Krieg, A.M. (eds.) 1988. Applied Antisense Oligonucleotide Technology. WileyLiss, New York.

Tamura, Y., Miyoshi, L.H., Yokota, T., and Makino, K. 1998. Preparation of stereoregulated antisense oligodeoxyribonucleoside phosphorothioate and interaction with its complementary DNA and RNA. Nucleosides Nucleotides 17:269-282.

Tang, J., Roskey, A., Li, Y., and Agrawal, S. 1995. Enzymatic synthesis of stereoregular [all $\mathrm{R}_{\mathrm{P}}$ ]oligonucleotide phosphorothioate and its properties. Nucleosides Nucleotides 14:985-990.

Thuong, N.T. and Helene, C. 1993. Sequence-specific recognition and modification of doublehelical DNA by oligonucleotides. Angew. Chem. Int. Ed. Engl. 32:666-690.

Wada, T., Kobayashi, N., Mori, T., and Sekine, M. 1998. Stereocontrolled synthesis of dithymidine phosphorothioates by use of a functionalized 5 '-protecting group bearing an imidazole residue. Nucleosides Nucleotides 17:351-364.

Wang, J.C. and Just, G. 1999. Indol-oxazaphosphorine precursors for stereoselective synthesis of dinucleotide phosphorothioates. J. Org. Chem. 64:8090-8097.

Wickstrom, E. 1986. Oligodeoxynucleotide stability in subcellular extracts and culture media. $J$. Biochem. Biophys. Methods 13:97-102.
Synthesis of Modified Oligonucleotides and Conjugates 4.17.27

Supplement 14 
Wilk, A. and Stec, W.J. 1995. Analysis of oligo(deoxynucleoside phosphorothioate)s and their diastereomeric composition. Nucl. Acids Res. 23:530-534.

Wilk, A., Grajkowski, A., Phillips, L.R., and Beaucage, S.L. 2000. Deoxyribonucleoside cyclic Nacylphosphoramidites as a new class of monomers for the stereocontrolled synthesis of oligothymidylyl- and oligodeoxycytidylylphosphorothioates. J. Am. Chem. Soc. 122:21492156.

Willson, M., Goncalves, H., Boudjebel, H., and Burgada, R. 1975. Étude du méchanisme des réactions d'acidalyse, d'alcoolyse et d'aminolyse des oxathiaphosphalanes-1,3,2. Bull. Soc. Chim. Fr. Part 2, 615-620.
Zon, G., Summers, M.F., Gallo, K.A., Shao, K.-L., Koziolkiewicz, M., Uznanski, B., and Stec, W.J. 1987. Stereochemistry of oligodeoxyribonucleotide phosphate triesters. In Biophosphates and Their Analogues: Synthesis, Structure, Metabolism and Activity (K.S. Bruzik and W.J. Stec, eds.) pp. 165-178. Elsevier, Amsterdam.

Contributed by Piotr Guga and

Wojciech J. Stec

Polish Academy of Sciences

Lodz, Poland 